\title{
Alma Imaging of Hcn, Cs, and Dust in Arp 220 and Ngc 6240
}

\section{Citation}

Scoville, Nick, Kartik Sheth, Fabian Walter, Swarnima Manohar, Laura Zschaechner, Min Yun, Jin Koda, et al. 2015. "ALMA IMAGING OF HCN, CS, AND DUST IN ARP 220 AND NGC 6240." The Astrophysical Journal 800 (1): 70. https://doi.org/10.1088/0004-637x/800/1/70.

\section{Permanent link}

http://nrs.harvard.edu/urn-3:HUL.InstRepos:41381713

\section{Terms of Use}

This article was downloaded from Harvard University's DASH repository, and is made available under the terms and conditions applicable to Other Posted Material, as set forth at http:// nrs.harvard.edu/urn-3:HUL.InstRepos:dash.current.terms-of-use\#LAA

\section{Share Your Story}

The Harvard community has made this article openly available.

Please share how this access benefits you. Submit a story.

Accessibility 


\title{
ALMA IMAGING OF HCN, CS, AND DUST IN ARP 220 AND NGC 6240
}

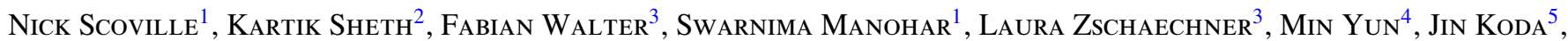 \\ David SAnders $^{6}$, Lena Murchikova ${ }^{1}$, Todd Thompson ${ }^{7,8}$, Brant Robertson ${ }^{9}$, Reinhard Genzel ${ }^{10}$, Lars HernQuist ${ }^{11}$, \\ Linda Tacconi $^{9}$, Robert Brown ${ }^{12}$, Desika Narayanan ${ }^{9}$, Christopher C. HayWard $^{13}$, Joshua Barnes ${ }^{6}$, \\ Jeyhan Kartaltepe ${ }^{14}$, Richard Davies ${ }^{10}$, Paul van Der WerF ${ }^{15}$, AND Edward Fomalont ${ }^{2,12}$ \\ ${ }^{1}$ California Institute of Technology, MC 249-17, 1200 East California Boulevard, Pasadena, CA 91125, USA \\ 2 North American ALMA Science Center, National Radio Astronomy Observatory, 520 Edgemont Road, Charlottesville, VA 22901, USA \\ ${ }^{3}$ Max-Planck-Institut fur Astronomie, Konigstuhl 17, D-69117 Heidelberg, Germany \\ ${ }^{4}$ Department of Astronomy, University of Massachusetts, Amherst, MA 01003, USA \\ ${ }^{5}$ Department of Physics and Astronomy, Stony Brook University, Stony Brook, NY 11794, USA \\ ${ }^{6}$ Institute for Astronomy, 2680 Woodlawn Drive, University of Hawaii, Honolulu, Hawaii, HI 96822, USA \\ ${ }^{7}$ Department of Astronomy, The Ohio State University, 140 West 18th Avenue, Columbus, OH 43210, USA \\ ${ }^{8}$ Center for Cosmology and AstroParticle Physics, The Ohio State University, 191 West Woodruff Avenue, Columbus, OH 43210, USA \\ ${ }^{9}$ Department of Astronomy and Steward Observatory, University of Arizona, Tucson AZ 85721, USA \\ ${ }^{10}$ Max-Planck-Institut fur extraterrestrische Physik (MPE), Giessenbachstrasse, D-85748 Garching, Germany \\ ${ }_{11}^{11}$ Harvard-Smithsonian Center for Astrophysics, 60 Garden Street, Cambridge, MA 02138, USA \\ 12 National Radio Astronomy Observatory, 520 Edgemont Road, Charlottesville, VA 22901, USA \\ ${ }^{13}$ TAPIR 350-17, California Institute of Technology, 1200 East California Boulevard, Pasadena, CA 91125, USA \\ ${ }^{14}$ National Optical Astronomy Observatory, 950 North Cherry Avenue, Tucson, AZ 85719, USA \\ ${ }^{15}$ Leiden Observatory, Leiden University, P.O. Box 9513, NL-2300 RA Leiden, The Netherlands \\ Received 2014 October 2; accepted 2014 December 15; published 2015 February 10
}

\begin{abstract}

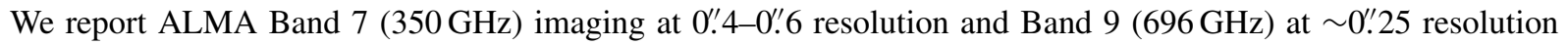
of the luminous IR galaxies Arp 220 and NGC 6240. The long wavelength dust continuum is used to estimate interstellar medium masses for Arp 220 east and west and NGC 6240 of 1.9, 4.2, and $1.6 \times 10^{9} M_{\odot}$ within radii of 69 , 65 , and $190 \mathrm{pc}$. The HCN emission was modeled to derive the emissivity distribution as a function of radius and the kinematics of each nuclear disk, yielding dynamical masses consistent with the masses and sizes derived from the dust emission. In Arp 220, the major dust and gas concentrations are at radii less than 50 pc in both counter-rotating nuclear disks. The thickness of the disks in Arp 220 estimated from the velocity dispersion and rotation velocities are $10-20 \mathrm{pc}$ and the mean gas densities are $n_{\mathrm{H}_{2}} \sim 10^{5} \mathrm{~cm}^{-3}$ at $R<50 \mathrm{pc}$. We develop an analytic treatment for the molecular excitation (including photon trapping), yielding volume densities for both the HCN and CS emission with $n_{H 2} \sim 2 \times 10^{5} \mathrm{~cm}^{-3}$. The agreement of the mean density from the total mass and size with that required for excitation suggests that the volume is essentially filled with dense gas, i.e., it is not cloudy or like swiss cheese.
\end{abstract}

Key words: galaxies: individual (Arp 220, NGC 6240) - galaxies: starburst

\section{INTRODUCTION}

Numerous multi-wavelength observational and theoretical investigations have revealed the basic properties and evolutionary scenario of ultraluminous infrared galaxies (ULIRGs). The first complete sample for the local universe was generated from the IRAS all sky survey, consisting of 12 galaxies with $L_{1-1000 \mu \mathrm{m}}>10^{12} L_{\odot}$ at $z<0.1$, and follow-up groundbased optical imaging revealed virtually all of the sample to be merging galaxies or post merging systems (Sanders et al. 1988; Sanders \& Mirabel 1996). All are also gas-rich with molecular gas masses determined from CO to be $2-20 \times 10^{9} M_{\odot}$ (Sanders et al. 1991). For the ULIRGs, approximately equal numbers of galaxies have optical emission line ratios characteristic of star formation (SF) and active galactic nuclei (AGNs; Sanders et al. 1988). Although the ULIRG activity is rare at low redshift, these merging systems are likely much more prevalent during the epoch of peak cosmic SF and nuclear activity at $z \sim 1-4$ (Le Floc'h et al. 2005).

$\operatorname{Arp} 220\left(L_{I R}=2.5 \times 10^{12} L_{\odot}, D_{L}=77 \mathrm{Mpc}, D_{A}=74 \mathrm{Mpc}\right.$, $\left.361 \mathrm{pc} \mathrm{arcsec}^{-1}\right)$ and NGC $6240\left(L_{I R}=0.9 \times 10^{12} L_{\odot}, D_{L}=\right.$ $103 \mathrm{Mpc}, D_{A}=98 \mathrm{Mpc}, 475 \mathrm{pc} \operatorname{arcsec}^{-1}$ ) are two of the most studied luminous IR galaxies. Both systems show double nuclei, separated by 1..0 (361 pc, Arp 220) and 1".5 (713 pc, NGC 6240). In Arp 220 the star formation rate (SFR) must be $\sim 240 M_{\odot} \mathrm{yr}^{-1}$ if the infrared luminosity is powered by SF. In NGC 6240 both galactic nuclei show X-ray emission suggesting that AGN accretion contributes some of the luminosity (Komossa et al. 2003). In NGC 6240 the molecular gas peak lies between the two NIR and X-ray nuclei so it is likely that most of the IR luminosity originates from star formation (SFR $\sim 70 M_{\odot} \mathrm{yr}^{-1}$ ) rather than AGN accretion on the nuclei.

During the merging of gas-rich galaxies, the original interstellar medium (ISM) (presumably distributed in extended galacticscale disks) sinks rapidly to the center of the merging system due to dissipation of kinetic energy in the shocked gas and torques generated by the offset stellar and gaseous bars (e.g., Barnes \& Hernquist 1992). The SFRs in the ULIRGs are typically 10-100 times higher per unit mass of ISM compared to quiescent disk galaxies. The ULIRG-starburst activity is likely driven by the concentration of gas in the nuclear regions and dynamical compression of this gas by supersonic shocks. Arp 220 has two nuclei separated by $\sim 300$ pc and over $90 \%$ of its bolometric luminosity emerges in the infrared. The two nuclei have counter-rotating disks of molecular gas as traced by $\mathrm{CO}$ emission (Sakamoto et al. 1999). In NGC 6240, the molecular gas extends between the nuclei rather than being concentrated on the individual nuclei.

The observations presented here were made with the ALMA array at 350 and $700 \mathrm{GHz}$ (Bands 7 and 9). These data provide 
excellent resolution and sensitivity for imaging high excitation molecular gas tracers ( $\mathrm{HCN}$ and CS) together with the optically thin long wavelength dust continuum. The dust continuum provides an independent and linear probe of the overall ISM mass (Scoville et al. 2014).

\subsection{Arp 220: Summary of Prior Observations}

Near infrared imaging of Arp 220 reveals two nuclei separated by 1 ; ; the west nucleus shows greatly increased extinction to the south, modeled as a dense dust disk tilted to the line of sight (Scoville et al. 1998). CO imaging at 0.'5 resolution detects the counter-rotating disks in both nuclei with radii $\sim 100$ pc, dynamical masses of $2 \times 10^{9} M_{\odot}$ for each disk and $\sim 50 \%$ gas mass fractions (Scoville et al. 1997; Sakamoto et al. 1999; Downes \& Eckart 2007). The inferred visible extinctions perpendicular to the disks are $\mathrm{A}_{V} \sim 500-2000 \mathrm{mag}$, precluding the use of optical/near infrared tracers of the SF in the nuclei. Near infrared integral field spectroscopy of the stars at larger radii indicates a significant population with age only $10 \mathrm{Myr}$ (Engel et al. 2011). Much of the nuclear disk ISM is very dense $\left(>10^{4-5} \mathrm{~cm}^{-3}\right)$ and at high temperatures $(T>75 \mathrm{~K})$ compared to normal Galactic molecular gas. This is based on the strong $\mathrm{HCN}$ and $\mathrm{HCO}^{+}$line emission observed with single dish telescopes and the high $\mathrm{CO}$ brightness and dust temperatures 75-170 K (Matsushita et al. 2009; Greve et al. 2009; Sakamoto et al. 1999; Downes \& Eckart 2007; Rangwala et al. 2011; Wilson et al. 2014). PCygni absorption line profiles are seen in $\mathrm{HCO}^{+}(J=3-2$ and 4-3) with Submillimeter Array (SMA), which Sakamoto et al. (2009) interpret as an outflow from the inner radii of the nuclear disks. HNC $(J=3-2)$ emission has also been imaged at 0 .' 3 resolution with SMA, but the emission is bright only in the western nucleus (Aalto et al. 2009).

\subsection{NGC 6240: Summary of Prior Observations}

In the infrared, NGC 6240 shows two nuclei separated by $\sim 2 \operatorname{arcsec}(700 \mathrm{pc})$ with high reddening in the region between them (based on the 2.2/1.6 $\mu \mathrm{m}$ color gradients; Scoville et al. 2000). Each of the nuclei exhibits X-ray emission suggestive of supermassive black hole accretion (Komossa et al. 2003). High-resolution CO $(J=2-1)$ imaging shows the molecular gas peaking in this "overlap region" (Bryant \& Scoville 1999; Tacconi et al. 1999; Engel et al. 2010). The gas concentration between the nuclei can be modeled as a rotating disk with dynamical mass $7 \times 10^{9} M_{\odot}$ and a molecular gas mass fraction of $\sim 50 \%$ (Tacconi et al. 1999). Iono et al. (2007) finds the CO (3-2) emission extended on a scale of $4 \mathrm{kpc}$ but the $\mathrm{HCO}^{+}(4-3)$ emission is concentrated between the two nuclei. Near infrared spectroscopy of NGC 6240 indicates a significant starburst (SB) population less than 20 Myr old (Tecza et al. 2000; Engel et al. 2010).

\subsection{Probing the Nuclear Structure}

Although the global understanding of merger evolution is well developed, major aspects of the structure and evolution of the massive gas-rich nuclear disks remain poorly constrained by observations and only crudely understood in terms of the physical processes. Specifically, the radial mass and SF distributions, the physical conditions (density, temperature, and cloud structures), and the dynamical evolution of the disks (due to accretion and feedback from SB and AGN activities) are undetermined. However, all of these are vital to understanding the final evolution of the mergers and the resulting SB properties (SF efficiency, distribution, and feedback), AGN fueling and the feedback of processed material to the circum-galactic environment.

Here, we present ALMA imaging at $350 \mathrm{GHz}(850 \mu \mathrm{m})$ of Arp 220 and NGC 6240 at $0.3-0$ '. 6 resolution. The observations include both the dust continuum, which is optically thin at long wavelengths and the emission lines of high excitation molecular gas tracers ( $\mathrm{HCN}, \mathrm{CS}$, and $\mathrm{CN}$ ). The $\mathrm{HCN}$ and $\mathrm{CS}$ emissions trace higher excitation molecular gas than is probed by the $\mathrm{CO}$ lines, since they require high column densities (and/or volume densities) to maintain population in the excited levels from which the emission originates. Thus, they provide better access to the gas closest to the centers of SB and AGN activity (as opposed to the extended molecular gas producing the $\mathrm{CO}$ emission).

We describe the observations and data reduction in Section 2 and summarize the results in Section 3. In Section 4, we use the dust emission to estimate the masses of ISM in each source. In Section 5 and the Appendix, we model the nuclear disk dynamics and HCN emissivity distributions in Arp 220 and NGC 6240, doing a maximum likelihood fit to obtain a best match to the observed HCN emission line profiles. This dynamical modeling yields total masses consistent with those derived from the dust continuum emission.

\section{ALMA OBSERVATIONS AND DATA REDUCTION}

The ALMA Cycle 0 observations for project 2011.0.00175.S were obtained in 2012. For both B and 7 and Band 9 observations, the correlator was configured in the time division mode with four spectral windows. Each window had a full bandwidth of $1875 \mathrm{MHz}$ covered by $31.25 \mathrm{MHz}$ resolution spectral channels. Three of the four windows in Band 7 were configured to observe spectral lines at rest frequencies of 342.882 (CS 7-6) and $354.505 \mathrm{GHz}$ ( $\mathrm{HCN} J=4-3$ ), while the fourth spectral window was centered at $340.974 \mathrm{GHz}$ to image the dust continuum emission. For the Band 9 observations, we configured the correlator to map two spectral lines at rest frequencies of 708.877 ( $\mathrm{HCN} \mathrm{8-7)}$ and $713.341 \mathrm{GHz}\left(\mathrm{HCO}^{+} 8-7\right)$ and used the two remaining spectral windows to image the continuum emission; these latter windows were centered at rest frequencies 707.3 and $711.0 \mathrm{GHz}$. The adopted redshifts were $z=0.01812$ and 0.02448 for Arp 220 and NGC 6240, respectively. For Band 9, we only present the continuum data here. (The Band 9 line emission data are not discussed further here and will be presented in a separate publication.)

All observations were done in the most extended configuration available in Cycle 0. For this telescope configuration, good flux recovery is expected out to scales of $\sim 3^{\prime \prime}$ and more extended emission will be partially resolved out. The spatial scales of the high excitation gas is confined to $<3^{\prime \prime}$ and observations with the ALMA compact telescope configuration were not necessary. The $\mathrm{CO}$ and dust continuum emission from the larger galaxies is likely to be significantly resolved out at $3^{\prime \prime}$ scales (Scoville et al. 1997). Most of the data were taken with configurations of 16-21 antennas, except one execution block that had 25 antennas. The total integration time for the Band 7 observations for NGC 6240 and Arp 220 was 140 minutes for each source (excluding calibrations), whereas for the Band 9 observations, the total on-source time was 32 minutes for Arp 220 and 75 minutes for NGC 6240.

Following the delivery of data products, the data were rereduced and imaged using the Common Astronomy Software Applications package. Modest self-calibration was done to improve the dynamic range. We measured the noise from the 
emission free regions of the map before subtraction of the continuum. The $1 \sigma$ (rms) sensitivities are as follows: for Arp 220 in Band 7, we measure an rms noise of $1.1 \mathrm{mJy} \mathrm{beam}^{-1}$ in $13 \mathrm{~km} \mathrm{~s}^{-1}$ channels in the upper sideband spectral windows (i.e., at a rest frequency of $\sim 354 \mathrm{GHz}$ ) and $1.7 \mathrm{mJy} \mathrm{beam}^{-1}$ in $13 \mathrm{~km} \mathrm{~s}^{-1}$ channels in the lower sideband (LSB) spectral windows (i.e., at a rest frequency of $342 \mathrm{GHz}$ ). For NGC 6240 we have a noise level of $\sigma \simeq 0.5 \mathrm{mJy} \mathrm{beam}^{-1}$ and $0.6 \mathrm{mJy} \mathrm{beam}^{-1}$ in the upper and LSBs in $13 \mathrm{~km} \mathrm{~s}^{-1}$ channels. The poorer sensitivity in the Arp 220 observations is largely due to the lower elevation of Arp 220 and shorter integration times.

Both the delivered data products and our re-reduction of the Arp 220 data sets exhibited non-flat residuals in line-free portions of the spectra after subtraction of the continuum in the UV data. After attempting to use the much weaker phase calibrator for passband calibration, we chose to use the original passband calibrator and then did continuum subtraction in the image plane. This was done in IDL using our own software, which fit a polynomial to line-free frequency planes for each spatial pixel, and then the endpoints of these polynomials were used to linearly interpolate across the frequency planes with possible line emission.

\section{OBSERVATIONAL RESULTS}

In this section, we present the results for both sources, first the continuum (Section 3.1) and then the line imaging (Section 3.2), followed by source flux measurements (Section 3.3). The source measurements are made by integration over circular apertures (Table 1) and by fitting multiple Gaussian components (Table 2). Synthesized beam parameters are given in Table 3. For Band 7 , the typical beam size is 0.5 and for Band 9 they are 0.3 (see Table 3). For the imaging we use coordinate offsets from Arp 220 west, i.e., the Gaussian component fit to the $698 \mathrm{GHz}$ continuum $\left(\alpha_{2000}=15: 34: 57.217\right.$ and $\left.\delta_{2000}=23: 30: 11.44\right)$ and for NGC 6240, the Gaussian-fit continuum peak at $694 \mathrm{GHz}$ $\left(\alpha_{2000}=16: 52: 58.898\right.$ and $\left.\delta_{2000}=2: 24: 03.51\right)$.

\subsection{Continuum}

At submillimeter wavelengths the continuum in ULIRGs is dominated by dust emission, specifically the Rayleigh-Jeans (RJ) tail of the far-infrared dust emission. The total fluxes measured for Arp 220 and NGC 6240 are 490 and $20 \mathrm{mJy}$ at $345 \mathrm{GHz}$ and 4.51 and $0.126 \mathrm{Jy}$ at $698 \mathrm{GHz}$ (Table 1 ). The synchrotron and free-free emission that is responsible for the longer wavelength radio continuum is $<30$ and $<16 \mathrm{mJy}$ at $v=$ $85 \mathrm{GHz}$ in Arp 220 and NGC 6240, respectively (Imanishi et al. 2007; Nakanishi et al. 2005). Of course, both the synchrotron and free-free emissions decrease at higher frequency (varying as $\sim v^{-0.7}$ and $v^{-0.1}$, respectively). Their contributions to the fluxes observed here are therefore negligible. The submillimeterwavelength dust emission is generally optically thin and the observed RJ continuum fluxes can be used to probe the ISM mass distributions (Scoville et al. 2014). In Section 4 we use the measured fluxes to estimate the ISM masses for each galaxy nucleus.

The continuum images of Arp 220 and NGC 6240 at $345 \mathrm{GHz}$ (Band 7) and $698 \mathrm{GHz}$ (Band 9) are shown in Figure 1. The image in Figure 1 (lower left) is similar in angular resolution and frequency to that recently obtained by Wilson et al. (2014) with ALMA. The $342 \mathrm{GHz}$ image (upper left) has $2 \times$ lower angular resolution but much higher signal-to-noise ratio $(\mathrm{S} / \mathrm{N})$ than the $350 \mathrm{GHz}$ SMA map of Sakamoto et al. (2008).
Table 1

Aperture Measurements

\begin{tabular}{|c|c|c|c|c|}
\hline Source & Apert. $^{\mathrm{a}}$ & \multicolumn{3}{|c|}{ Total $^{\text {b }}$} \\
\hline \multicolumn{5}{|c|}{ Lines } \\
\hline & $" 1$ & & $\mathrm{Jy} \mathrm{km} \mathrm{s}^{-1}$ & \\
\hline Arp 220 total & 1.8 & $\mathrm{HCN}(4-3)$ & $331 \pm 30$ & \\
\hline Arp 220 east & 1.2 & $\mathrm{HCN}(4-3)$ & $106 \pm 11$ & \\
\hline Arp 220 west & 1.2 & $\mathrm{HCN}(4-3)$ & $231 \pm 23$ & \\
\hline Arp 220 total & 1.8 & CS (7-6) & $62 \pm 6$ & \\
\hline Arp 220 east & 1.2 & CS (7-6) & $24 \pm 2$ & \\
\hline Arp 220 west & 1.2 & CS (7-6) & $42 \pm 4$ & \\
\hline NGC 6240 & 3.0 & $\mathrm{CN}$ & $>39^{c}$ & \\
\hline NGC 6240 & 3.0 & CS (7-6) & $7.4 \pm 1$ & \\
\hline NGC 6240 & 3.0 & $\mathrm{HCN}(4-3)$ & $36.9 \pm 4$ & \\
\hline \multicolumn{5}{|c|}{ Continuum } \\
\hline & & $\mathrm{GHz}$ & Jy & Jy beam $^{-1}$ \\
\hline \multicolumn{5}{|c|}{ Band 7} \\
\hline Arp 220 total & 1.8 & 335.9 & $0.458 \pm 0.012$ & $0.234 \pm 0.03$ \\
\hline Arp 220 total & 1.8 & 347.6 & $0.490 \pm 0.002$ & $0.288 \pm 0.01$ \\
\hline Arp 220 east & 1.2 & 335.9 & $0.152 \pm 0.017$ & $0.106 \pm 0.04$ \\
\hline Arp 220 east & 1.2 & 347.6 & $0.161 \pm 0.017$ & $0.119 \pm 0.02$ \\
\hline Arp 220 west & 1.2 & 335.9 & $0.313 \pm 0.007$ & $0.234 \pm 0.03$ \\
\hline Arp 220 west & 1.2 & 347.6 & $0.342 \pm 0.002$ & $0.288 \pm 0.01$ \\
\hline NGC 6240 & 3.0 & 333.9 & $0.024 \pm 0.002$ & $0.008 \pm 0.01$ \\
\hline NGC 6240 & 3.0 & 345.3 & $0.018 \pm 0.002$ & $0.006 \pm 0.01$ \\
\hline \multicolumn{5}{|c|}{ Band 9} \\
\hline Arp 220 total & 1.8 & 697.7 & $4.14 \pm 0.07$ & $2.75 \pm 0.02$ \\
\hline Arp 220 east & 0.8 & 697.7 & $2.15 \pm 0.15$ & $1.36 \pm 0.06$ \\
\hline Arp 220 west & 0.8 & 697.7 & $3.80 \pm 0.04$ & $2.75 \pm 0.03$ \\
\hline NGC 6240 & 3.0 & 693.5 & $0.106 \pm 0.004$ & $0.025 \pm 0.01$ \\
\hline
\end{tabular}

Notes. Total integrated fluxes were measured in circular apertures of diameter given in Column 2. The peak fluxes were measured with the synthesized beams given in Table 3 but restricted to the same diameter regions.

${ }^{\text {a }}$ Measurement aperture diameter in arcseconds.

${ }^{\mathrm{b}}$ Uncertainties on the line fluxes are taken to be the larger of the $10 \%$ uncertainty in flux calibration or the measured rms noise. The uncertainties for the continuum measurements were derived from the dispersion in measurements taken in equal size apertures centered at off-source positions.

${ }^{\mathrm{c}}$ Lower limit since the line is on the edge of the bandpass.

Since the long wavelength dust emission is optically thin, the centroid of the dust emission may be used to pinpoint the ISM center of mass in the galaxy nucleus. In Figure 2 we show the $698 \mathrm{GHz}$ continuum contours together with the nuclear emission peaks found in previous high-resolution imaging: the $33 \mathrm{GHz}$ radio continuum (Barcos-Muñoz et al. 2014), the $349 \mathrm{GHz}$ continuum (Sakamoto et al. 2008), and the $691 \mathrm{GHz}$ continuum (Wilson et al. 2014). The peak positions obtained here (the plus and diamond symbols in Figure 2) were derived from the Gaussian-fit components as were those from Sakamoto et al. (2008) and Wilson et al. (2014). The peak positions all agree to within 0 '.2. The small shifts in the submillimeter peaks, shown in Figure 2, could be caused by depth effects for the dust emission, or they may simply reflect uncertainties in the interferometric phase calibrations for Cycle 0 ALMA at high frequencies. In the following, we adopt our $698 \mathrm{GHz}$ Gaussian-fit peaks (Table 2) as the best positions for the two galaxy nuclei.

The total continuum fluxes at $350 \mathrm{GHz}(870 \mu \mathrm{m})$ and $700 \mathrm{GHz}(430 \mu \mathrm{m})$ are: $0.490 \mathrm{Jy}$ and $4.51 \mathrm{Jy}$ for Arp 220 (Table 1). Single dish (JCMT-SCUBA) fluxes for Arp 220 are: $832 \pm 86$ and $455 \pm 47 \mathrm{mJy}$ (average $=643 \mathrm{mJy}$ ) at $850 \mu \mathrm{m}$ (Dunne et al. 2000; Antón et al. 2004) and 6.3 $\pm 0.8 \mathrm{Jy}$ at $450 \mu \mathrm{m}$ (Dunne \& Eales 2001). Taking these fluxes at "face value", our 
Table 2

Gaussian Fit Components

\begin{tabular}{|c|c|c|c|c|c|c|}
\hline Source & Line/Continuum & $\begin{array}{c}v_{\mathrm{obs}} \\
(\mathrm{GHz})\end{array}$ & $\alpha_{2000}$ & $\delta_{2000}$ & Peak Flux & $\begin{array}{c}\text { Peak } T_{B} \\
\quad(\mathrm{~K})\end{array}$ \\
\hline \multicolumn{7}{|c|}{ Band 7} \\
\hline & & & & & Jy beam $^{-1}$ & \\
\hline Arp $220 \mathrm{~W}$ & Continuum & 335.86 & $15: 34: 57.224$ & $23: 30: 11.357$ & $0.238 \pm 0.0007$ & 10.2 \\
\hline $\operatorname{Arp} 220 \mathrm{E}$ & Continuum & 335.86 & $15: 34: 57.286$ & $23: 30: 11.206$ & $0.105 \pm 0.0007$ & 4.5 \\
\hline Arp $220 \mathrm{~W}$ & Continuum & 347.60 & $15: 34: 57.224$ & $23: 30: 11.357$ & $0.293 \pm 0.0016$ & 14.6 \\
\hline Arp $220 \mathrm{E}$ & Continuum & 347.60 & $15: 34: 57.286$ & 23:30:11.199 & $0.118 \pm 0.0015$ & 5.9 \\
\hline NGC $6240 \mathrm{~S}$ & Continuum & 333.87 & $16: 52: 58.909$ & $2: 24: 03.203$ & $0.007 \pm 0.0003$ & 0.3 \\
\hline NGC $6240 \mathrm{~N}$ & Continuum & 333.87 & $16: 52: 58.916$ & $2: 24: 04.177$ & $0.003 \pm 0.0002$ & 0.1 \\
\hline NGC $6240 \mathrm{~S}$ & Continuum & 345.35 & $16: 52: 58.909$ & $2: 24: 03.200$ & $0.005 \pm 0.0001$ & 0.2 \\
\hline NGC $6240 \mathrm{~N}$ & Continuum & 345.35 & $16: 52: 58.920$ & $2: 24: 04.232$ & $0.002 \pm 0.0001$ & 0.1 \\
\hline \multicolumn{7}{|c|}{ Band 9} \\
\hline Arp $220 \mathrm{~W}$ & Continuum & 697.69 & $15: 34: 57.217$ & $23: 30: 11.440$ & $2.672 \pm 0.0053$ & 75.2 \\
\hline Arp 220 E & Continuum & 697.69 & $15: 34: 57.283$ & $23: 30: 11.282$ & $1.316 \pm 0.0048$ & 37.0 \\
\hline NGC 6240 & Continuum & 693.46 & $16: 52: 58.898$ & $2: 24: 3.512$ & $0.022 \pm 0.0001$ & 0.9 \\
\hline \multicolumn{7}{|c|}{ Lines } \\
\hline & & & & & $\mathrm{Jy} \mathrm{km} \mathrm{s}^{-1}$ beam $^{-1}$ & $\mathrm{~b}$ \\
\hline Arp $220 \mathrm{~W}$ & CS (7-6) & 336.71 & $15: 34: 57.219$ & $23: 30: 11.400$ & $24.730 \pm 0.0003$ & 4.73 \\
\hline Arp 220 E & CS (7-6) & 336.71 & $15: 34: 57.287$ & $23: 30: 11.221$ & $15.636 \pm 0.0003$ & 2.86 \\
\hline Arp $220 \mathrm{~W}$ & $\mathrm{HCN}(4-3)$ & 348.18 & $15: 34: 57.222$ & $23: 30: 11.383$ & $143.213 \pm 0.0005$ & 17.20 \\
\hline Arp $220 \mathrm{E}$ & $\mathrm{HCN}(4-3)$ & 348.18 & $15: 34: 57.288$ & $23: 30: 11.214$ & $56.775 \pm 0.0005$ & 10.30 \\
\hline NGC 6240 & CS (7-6) & 334.65 & $\ldots^{\mathrm{a}}$ & $\ldots^{\mathrm{a}}$ & $\ldots^{\mathrm{a}}$ & 0.27 \\
\hline NGC 6240 & $\mathrm{HCN}(4-3)$ & 345.99 & $16: 52: 58.907$ & $2: 24: 03.559$ & $8.985 \pm 0.0001$ & 1.10 \\
\hline
\end{tabular}

Notes. Peak position and fluxes obtained from two component 2D Gaussian fits. Gaussian component sizes are listed in Table 3. Uncertainties in the continuum and line fluxes obtained from the uncertainties in the Gaussian component fitting do not include calibration uncertainties.

${ }^{a}$ No convergent fit obtained.

${ }^{\mathrm{b}}$ Peak brightness temperature from the spectral cube within $\pm 500 \mathrm{~km} \mathrm{~s}^{-1}$ of the systemic velocity.

Table 3

Gaussian Source Sizes from Table 2

\begin{tabular}{|c|c|c|c|c|c|c|c|c|c|c|}
\hline \multirow[b]{2}{*}{ Source } & & \multicolumn{3}{|c|}{ Beam } & \multicolumn{3}{|c|}{ Source } & \multicolumn{3}{|c|}{ Deconvolved } \\
\hline & & $\begin{array}{c}\text { Major } \\
\left({ }^{\prime \prime}\right)\end{array}$ & $\begin{array}{c}\text { Minor } \\
\left({ }^{\prime \prime}\right)\end{array}$ & $\begin{array}{l}\text { P.A. } \\
\left({ }^{\circ}\right)\end{array}$ & $\begin{array}{c}\text { Major } \\
\left({ }^{\prime \prime}\right)\end{array}$ & $\begin{array}{c}\text { Minor } \\
\left({ }^{\prime \prime}\right)\end{array}$ & $\begin{array}{l}\text { P.A. } \\
\left(^{\circ}\right)\end{array}$ & $\begin{array}{c}\text { Major } \\
\left({ }^{\prime \prime}\right)\end{array}$ & $\begin{array}{c}\text { Minor } \\
\left({ }^{\prime \prime}\right)\end{array}$ & $\begin{array}{c}T_{B} \\
(\mathrm{~K})\end{array}$ \\
\hline & & \multicolumn{9}{|c|}{ Band 7} \\
\hline Arp $220 \mathrm{~W}$ & Continuum & 0.60 & 0.42 & -32.0 & $0.66 \pm 0.01$ & $0.50 \pm 0.01$ & $-29 \pm 1$ & 0.28 & 0.27 & 33.9 \\
\hline Arp 220 E & Continuum & 0.60 & 0.42 & -32.0 & $0.71 \pm 0.02$ & $0.50 \pm 0.01$ & $-16 \pm 1$ & 0.37 & 0.27 & 11.1 \\
\hline $\operatorname{Arp} 220 \mathrm{~W}$ & Continuum & 0.52 & 0.39 & -27.2 & $0.63 \pm 0.01$ & $0.46 \pm 0.02$ & $-28 \pm 1$ & 0.36 & 0.24 & 34.9 \\
\hline Arp $220 \mathrm{E}$ & Continuum & 0.52 & 0.39 & -27.2 & $0.65 \pm 0.01$ & $0.51 \pm 0.01$ & $-17 \pm 3$ & 0.38 & 0.32 & 9.6 \\
\hline NGC $6240 \mathrm{~S}$ & Continuum & 0.55 & 0.46 & 65.6 & $0.74 \pm 0.04$ & $0.60 \pm 0.03$ & $-152 \pm 13$ & 0.50 & 0.39 & 0.4 \\
\hline NGC $6240 \mathrm{~N}$ & Continuum & 0.55 & 0.46 & 65.6 & $1.22 \pm 0.11$ & $0.70 \pm 0.06$ & $-150 \pm 8$ & 1.09 & 0.53 & 0.1 \\
\hline NGC $6240 \mathrm{~S}$ & Continuum & 0.53 & 0.44 & 64.7 & $0.72 \pm 0.02$ & $0.59 \pm 0.02$ & $-155 \pm 2$ & 0.49 & 0.40 & 0.3 \\
\hline \multirow[t]{2}{*}{ NGC $6240 \mathrm{~N}$} & Continuum & 0.53 & 0.44 & 64.7 & $1.19 \pm 0.01$ & $0.72 \pm 0.02$ & $-149 \pm 1$ & 1.07 & 0.57 & 0.03 \\
\hline & & \multicolumn{9}{|c|}{ Band 9} \\
\hline Arp $220 \mathrm{~W}$ & Continuum & 0.32 & 0.28 & -38.6 & $0.39 \pm 0.01$ & $0.34 \pm 0.01$ & $-42 \pm 1$ & 0.23 & 0.19 & 148.9 \\
\hline Arp $220 \mathrm{E}$ & Continuum & 0.32 & 0.28 & -38.6 & $0.44 \pm 0.01$ & $0.37 \pm 0.02$ & $-139 \pm 1$ & 0.30 & 0.24 & 47.2 \\
\hline NGC 6240 & Continuum & 0.27 & 0.24 & 29.7 & $0.86 \pm 0.02$ & $0.39 \pm 0.02$ & $-173 \pm 1$ & 0.82 & 0.30 & 0.2 \\
\hline & & \multicolumn{9}{|c|}{ Lines } \\
\hline Arp $220 \mathrm{~W}$ & $\mathrm{CS}(7-6)$ & 0.60 & 0.42 & -32.0 & $0.78 \pm 0.02$ & $0.60 \pm 0.02$ & $-31 \pm 3$ & 0.49 & 0.43 & 10.1 \\
\hline Arp 220 E & CS (7-6) & 0.60 & 0.42 & -32.0 & $0.72 \pm 0.02$ & $0.54 \pm 0.02$ & $-35 \pm 2$ & 0.40 & 0.35 & 7.5 \\
\hline Arp $220 \mathrm{~W}$ & $\mathrm{HCN}(4-3)$ & 0.52 & 0.39 & -27.2 & $0.77 \pm 0.01$ & $0.57 \pm 0.01$ & $-24 \pm 1$ & 0.57 & 0.41 & 39.3 \\
\hline Arp $220 \mathrm{E}$ & $\mathrm{HCN}(4-3)$ & 0.52 & 0.39 & -27.2 & $0.78 \pm 0.01$ & $0.59 \pm 0.01$ & $20 \pm 2$ & 0.58 & 0.45 & 21.5 \\
\hline NGC 6240 & CS (7-6) & 0.55 & 0.46 & 65.6 & $\ldots^{\mathrm{a}}$ & $\ldots^{\mathrm{a}}$ & $\ldots^{\mathrm{a}}$ & $\ldots{ }^{a}$ & $\ldots^{\mathrm{a}}$ & $\ldots$ \\
\hline NGC 6240 & $\mathrm{HCN}(4-3)$ & 0.53 & 0.44 & 64.7 & $1.26 \pm 0.02$ & $0.74 \pm 0.02$ & $-178 \pm 3$ & 1.14 & 0.60 & 1.5 \\
\hline
\end{tabular}

Notes. Sizes and major axis P.A. estimates obtained from 2D Gaussian fits listed in Table 2. The uncertainties in the parameters were the formal errors from the multi-component Gaussian fitting.

${ }^{a}$ No convergent fit obtained. 

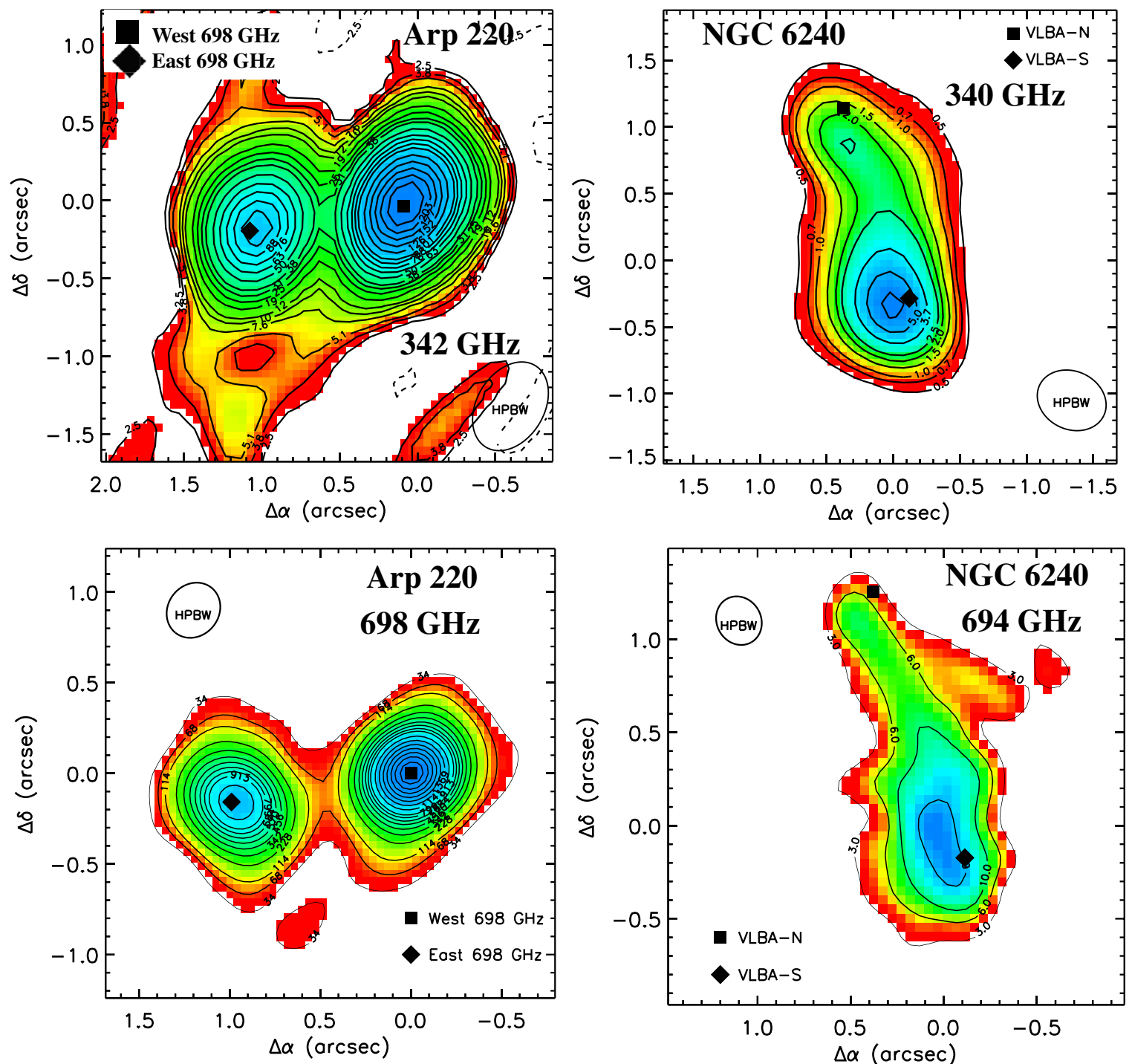

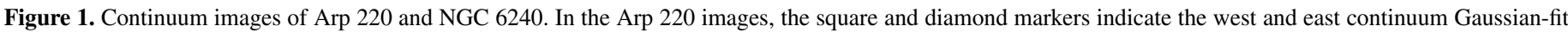

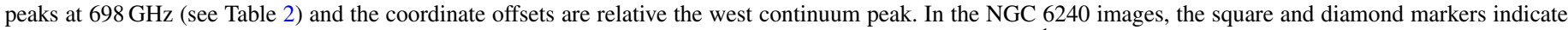

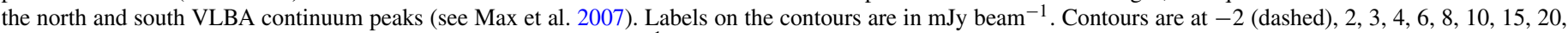

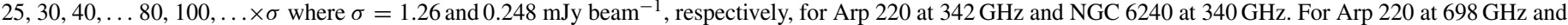

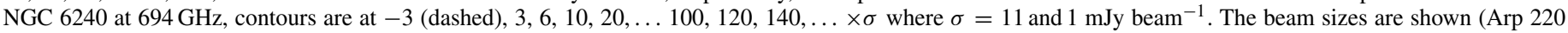
Band 7: 0.60-0.52 × 0.42-0.'39 and Band 9: 0.32 × $0^{\prime \prime} .28 ;$ NGC 6240 Band 7: $0.54 \times 045^{\prime \prime}$ and Band 9: 0.27 × 0'.24).

ALMA imaging is detecting approximately $76 \%$ and $71 \%$ of the total flux at 350 and $700 \mathrm{GHz}$. For NGC 6240 we obtain integrated fluxes of 18-24 and 126 mJy (Table 1), compared with the JCMT-SCUBA total fluxes of 0.15 and $1 \mathrm{Jy}( \pm 30 \%$; Klaas et al. 2001) at 850 and $450 \mu \mathrm{m}$. Here, the detected percentages are only $\sim 15 \%$. In NGC 6240 , the dust and line emission is clearly more extended than in Arp 220 so it is expected that the recovered flux percentage will be smaller than in Arp 220. However, the extremely small "apparent" percentage $(15 \%)$ in NGC 6240 is surprising since the telescope configuration should have good flux recovery for angular scales $<4$ ". It appears possible that there are significant calibration errors in the single dish measurements.

\subsection{Lines}

Figure 3 shows the source-integrated spectra for Arp 220 and NGC 6240 and Figure 4 shows the spectra for the individual east and west nuclei in Arp 220. The HCN (4-3) and CS (7-6) lines are seen at very high $\mathrm{S} / \mathrm{Ns}$ in all of the spectra with a similar ratio $\mathrm{HCN}(4-3) / \mathrm{CS}(7-6) \sim 0.2$ (see Table 1). The CN $(7 / 2-5 / 2)$ occurs at the low frequency end of the LSB and thus the redshifted side of the emission is truncated. In both nuclei of Arp 220 this line appears in emission and absorption at different velocities (see Figure 4). In NGC 6240 the CN emission is similar in strength to the HCN line (Figure 3).

The HCN and CS lines show double peaked velocity profiles in both nuclei of Arp 220 (Figure 4). This is possibly due to absorption by optically thick, lower excitation gas at the systemic velocity along the line of sight to the nuclei. Sakamoto et al. (2009) detect absorption in $\mathrm{HCO}^{+}(3-2$ and 4-3) and $\mathrm{CO}$ (3-2) in small apertures on both nuclei. The deepest absorption in their spectra occurs at $5200-5450 \mathrm{~km} \mathrm{~s}^{-1}$, corresponding to the high frequency side of the HCN and CS line profiles where the dip is seen. The $\mathrm{CN}(7 / 2-5 / 2)$ line shows strong absorption on both nuclei of Arp 220 but, since this line is near the edge of the bandpass, we will not discuss it further.

Figures 5 and 6 show the velocity-integrated line fluxes and mean velocities for HCN and CS in Arp 220 and NGC 6240. 


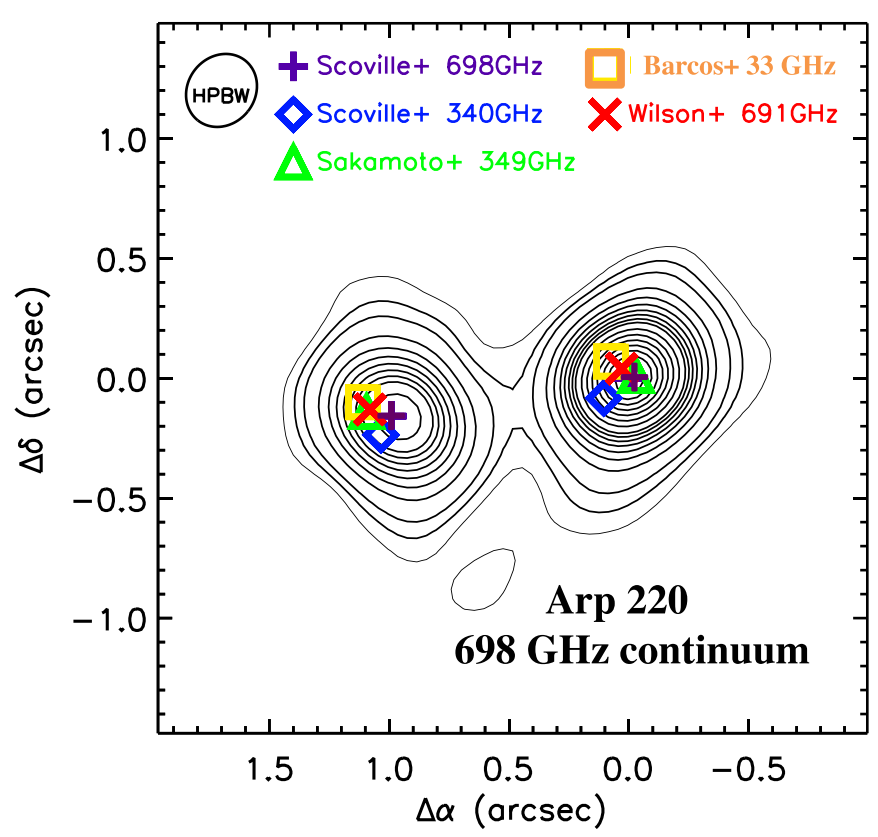

Figure 2. Positions of the two nuclei in Arp 220 as derived from high-resolution studies are compared: this work (Scoville et al.), Sakamoto et al. (2008), BarcosMuñoz et al. (2014), and Wilson et al. (2014). The contours represent our $698 \mathrm{GHz}$ continuum image (Figure 1). The position offsets are discussed in the text.

Both the integrated intensity and the mean velocities are calculated as simple moment integrals over $V= \pm 500 \mathrm{~km} \mathrm{~s}^{-1}$ (without a $\mathrm{S} / \mathrm{N}$ clipping threshold for the $3 \mathrm{~d}$ pixels). The velocities are measured relative to the adopted systemic redshifts $z=0.01812$ and 0.02448 . In both galaxies, the projected emissivity and velocity distributions are similar between $\mathrm{HCN}$ (4-3) and CS (7-6); the peaks coincide within 0 '. 1 and the sizes are similar (see Table 2).

In Arp 220 west, the apparent kinematic major axes for $\mathrm{HCN}$ and $\mathrm{CS}$ are misaligned by $\sim 45^{\circ}$ (P.A. $=-45^{\circ}$ for $\mathrm{HCN}$ and P.A. $\sim-90^{\circ}$ for CS, see Figure 5). Most of this disagreement is on the western side of the west nucleus where the CS intensities are low. Lastly, there is also a minor difference along the southern boundary of the nuclei; the east source showing a tongue of emission to the south in CS whereas in $\mathrm{HCN}$ a southerly extension is seen from the west nucleus (see Figure 5). Some of these differences may be due to the very different optical depths of the CS (7-6) and HCN (4-3) emission lines (see Figure 10, right).

In NGC 6240 both the HCN and CS lines are much weaker than in Arp 220 so the $\mathrm{S} / \mathrm{N}$ are not as high. In fact, the fitting of a single Gaussian component to the CS emission distribution did not converge due to low $\mathrm{S} / \mathrm{N}$ (see Table 2); however, a fit was obtained for $\mathrm{HCN}$. Comparing the relative placement of the ISM concentrations and the galaxy nuclei it is clear that in NGC 6240 the ISM is distributed along a ridge between the two nuclei (as located by the Very Long Baseline Array (VLBA) N and $\mathrm{S}$ sources and the IR nuclei in Max et al. 2007) rather than on the nuclei (as in Arp 220). This internuclear concentration of the molecular gas has been noted in previous investigations (Tacconi et al. 1999; Bryant \& Scoville 1999; Engel et al. 2010). However, the major peak of the submillimeter dust emission and the HCN and CS is clearly nearer the south nucleus (see Figures 1 and 6).

\subsection{Measurements and Source Components}

Aperture flux measurements for the continuum and the spectral lines are given in Table 1 . The table includes line fluxes integrated in velocity over $\pm 500 \mathrm{~km} \mathrm{~s}^{-1}$.

We also computed least squares fits of elongated Gaussians to the observed brightness distributions and the fit parameters are given in Tables 2 and 3. For Arp 220 both nuclei were fit simultaneously with two-dimensional Gaussians; the fits then provide a reasonable decomposition of the emission in the areas of overlap. In NGC 6240 a double Gaussians were simultaneously fit to the peak in the south plus the extended tail to the north. Table 3 includes an estimate of the true source sizes, with the beam analytically deconvolved. The peak brightness temperatures for both the observed and deconvolved emission distributions are given in Tables 2 and 3, respectively. For Arp 220 , the peak continuum deconvolved brightness temperatures reach $10-40 \mathrm{~K}$ in Band 7 and $50-150 \mathrm{~K}$ in Band 9. The blackbody temperature expected for the measured source size and luminosity is $T_{b b}=100 \mathrm{~K}$ (Section 4). It is likely that the dust emission is optically thick in Band 9 but optically thin in Band 7 (Downes \& Eckart 2007; Matsushita et al. 2009; Wilson et al. 2014).
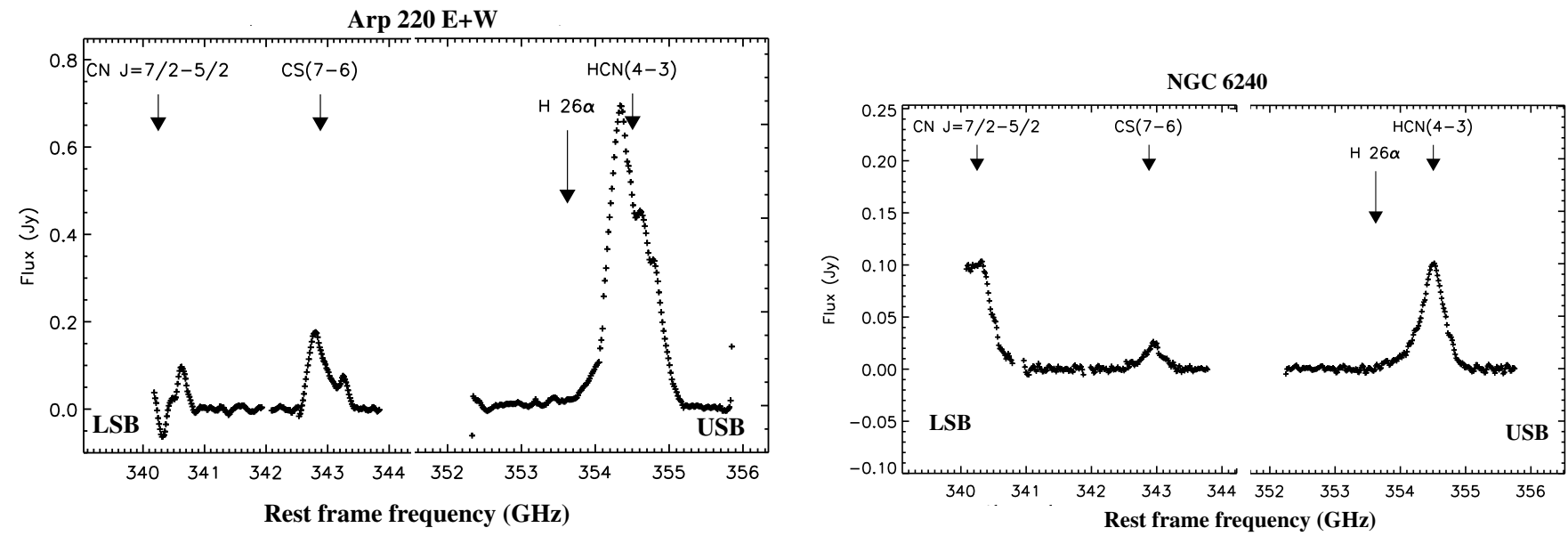

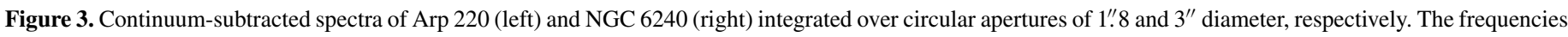

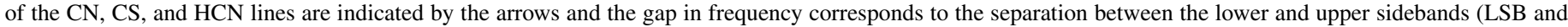

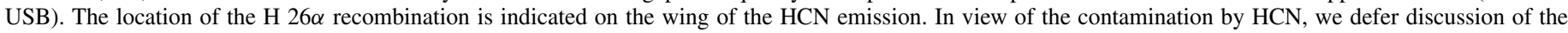
recombination lines (Scoville \& Murchikova 2013) to our scheduled ALMA Cycle 2 data, which includes other recombination lines. 

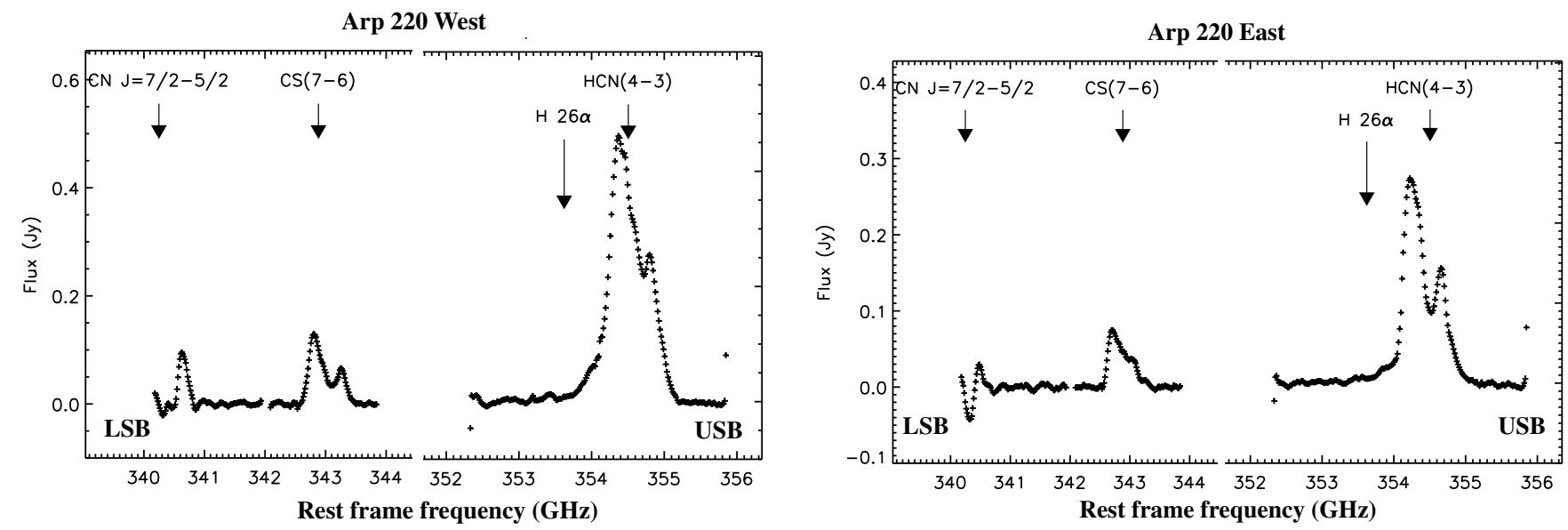

Figure 4. Continuum-subtracted spectra of the Arp 220 nuclei integrated over circular apertures of $1^{\prime \prime} .2$ diameter centered on each peak. The location of the H $26 \alpha$ recombination is indicated on the wing of the HCN emission. In view of the contamination by HCN, we defer discussion of the recombination lines (Scoville \& Murchikova 2013) to our scheduled ALMA Cycle 2 data, which includes other recombination lines.

Arp 220
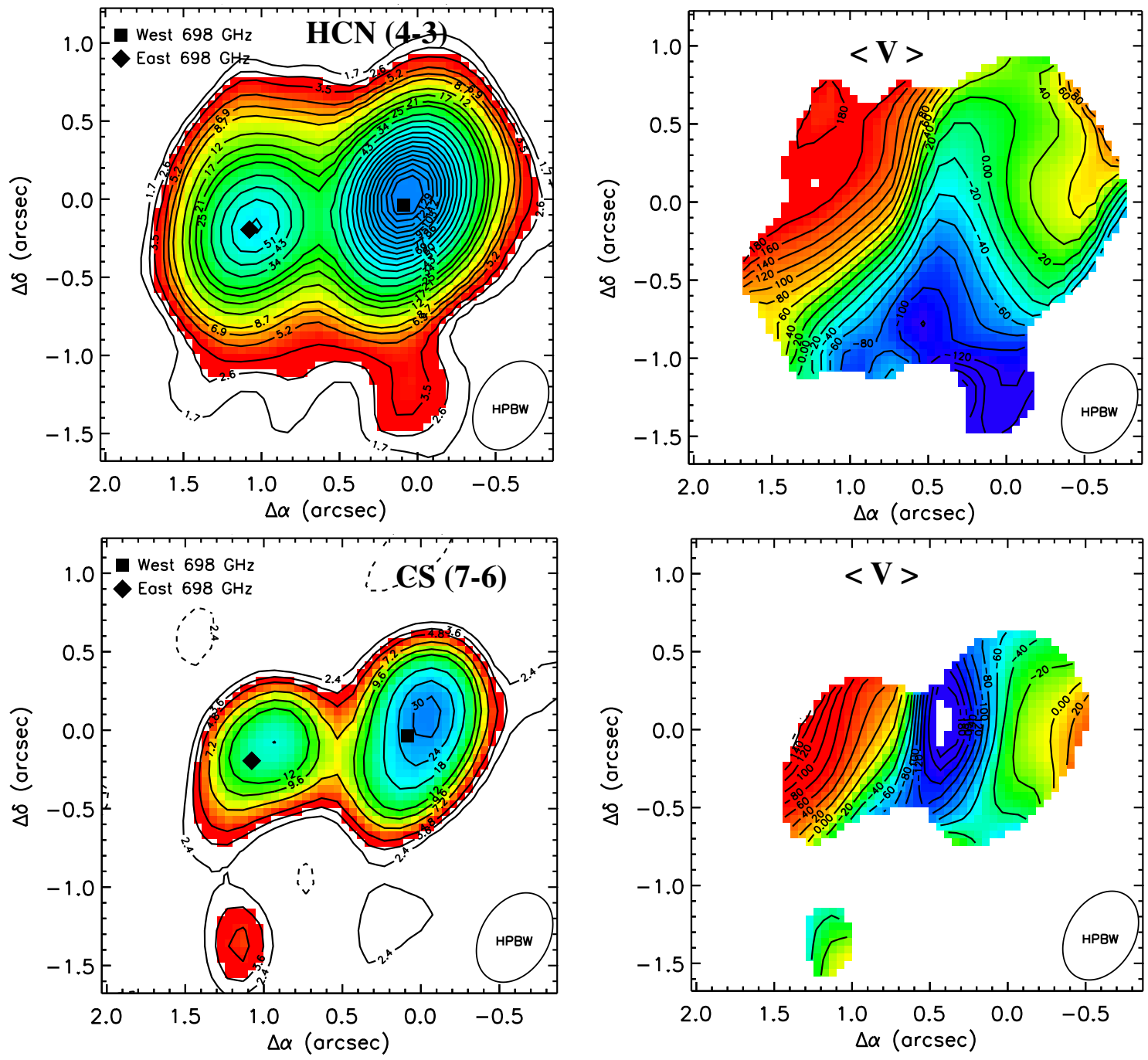

Figure 5. Arp 220: integrated line intensity images and mean velocities for the HCN (4-3) (top panels) and CS (7-6) emission (bottom panels). The square and diamond markers indicate the west and east continuum peaks at $698 \mathrm{GHz}$ (see Table 2) and the coordinate offsets are from the west continuum peak. Contours for $\mathrm{HCN}$ and CS are at -2 (dashed), 2, 3, 4, 6, 8, 10,15, 20, 25, 30, 40,50, . $\times \sigma$ where $\sigma=0.78$ and $1.2 \mathrm{Jy} \mathrm{beam}^{-1} \mathrm{~km} \mathrm{~s}^{-1}$. The velocity contour spacing is $20 \mathrm{~km} \mathrm{~s}^{-1}$ and the values are labeled. The beam sizes $(0.52 \times 0$ '” 38$)$ are shown. 


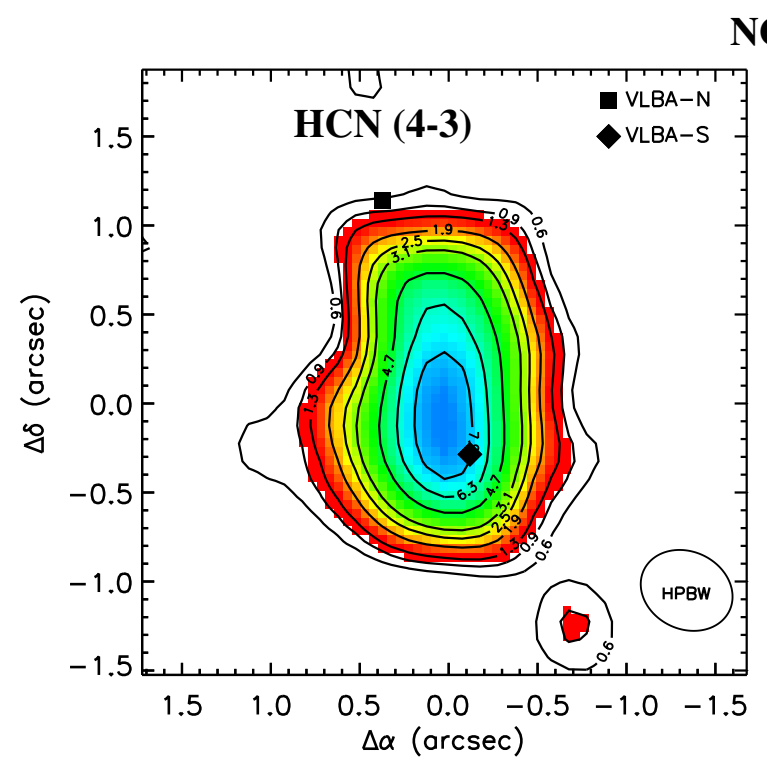

NGC 6240
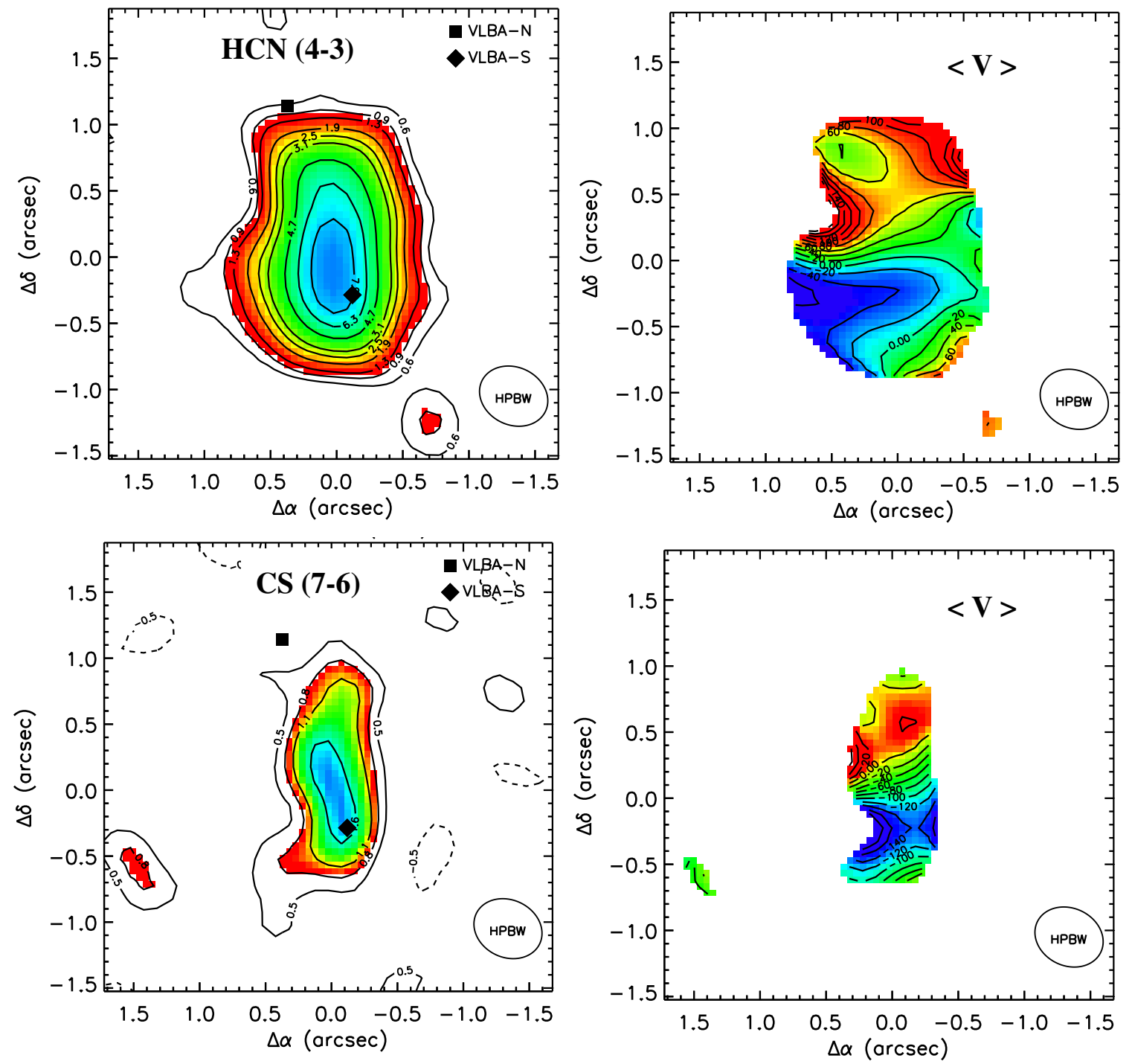

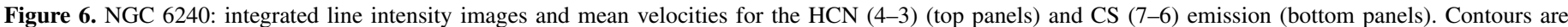

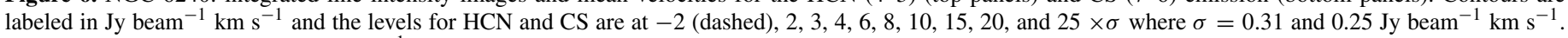
The velocity contour spacing is $20 \mathrm{~km} \mathrm{~s}^{-1}$ and the values are labeled. The beam sizes $\left(0.55 \times 0.0^{\prime \prime} 45\right)$ are shown.

The source sizes are probably best characterized by the dust continuum distribution using observations where the dust emission is optically thin, Band 7 for Arp 220 and Band 9 for NGC 6240. In Arp 220 west the deconvolved size is $0.36 \times 0$ 0'.24 (361 pc per"), implying a projected size of $130 \times 87 \mathrm{pc}$. For Arp 220 east, the size is $0.38 \times 0^{\prime \prime} .32$, or $137 \times 116 \mathrm{pc}$. If these are modeled as inclined disks the implied inclinations are $48^{\circ}$ and $31^{\circ}$ from these axis ratios (the deconvolved sizes are uncertain given that they are less than the observational resolution).

\section{LONG WAVELENGTH DUST CONTINUUM AS A MASS TRACER}

In this section we use the long wavelength dust emission to estimate the masses of dust and gas in each nucleus. Assuming the fluxes of the nuclei are well-recovered in the interferometry, this is a very robust mass estimator. At sufficiently long wavelengths the dust is optically thin so the observations sample the entire column of dust and ISM. The emitted flux on the RJ tail of the spectrum is only linearly dependent on the dust temperature $\left(T_{d}\right)$ and for normal galaxies the bulk of the ISM mass is at typically $20-40 \mathrm{~K}$. Averaging over whole galaxies or galactic nuclei, the dust opacity coefficient and dust-to-gas mass ratio are also probably not strongly varying (at least for massive galaxies with near solar metallicity). Thus the long wavelength dust continuum can be used as a linear probe of the ISM mass, avoiding the problems associated with optically thick molecular lines with sub-thermal excitation. In application of this technique to galactic nuclei, one does need to allow for increased dust temperatures in the stronger radiation field of the nuclear regions. The use of the dust continuum is fully developed along with empirical calibration of the flux to mass ratio in Scoville et al. (2014) and is summarized briefly below.

At long wavelengths, the dust emission is optically thin and the observed flux density is given by

$$
S_{v} \propto \kappa_{d}(v) T_{d} v^{2} \frac{M_{\mathrm{d}}}{d_{L}^{2}}
$$

where $T_{d}$ is the temperature of the grains, $\kappa_{d}(v)$ is the dust opacity per unit mass of dust, $M_{d}$ is the total mass of dust and 
$d_{L}$ is the luminosity distance. (For simplicity in illustrating the physics, Equation (1) does not include the effects of bandshifting and compression of frequency space that occur for high redshift sources.) In local star-forming galaxies, most of the mass of dust is at $T_{d} \sim 20 \rightarrow 30 \mathrm{~K}$ (see references in Scoville et al. 2014) and variations in the mass-weighted dust temperatures between galaxies are small $(\sim 20-40 \mathrm{~K})$; the observed fluxes then probe the total mass of dust.

To obviate the need to know both the dust opacity and dustto-gas ratio (which are degenerate when using Equation (1) to estimate ISM masses), one can instead empirically calibrate the ratio of the specific luminosity at rest frame $850 \mu \mathrm{m}$ to total ISM mass using samples of observed galaxies, thus absorbing the opacity curve and the abundance ratio into a single empirical constant $\alpha_{850 \mu \mathrm{m}}=L_{v_{850 \mu \mathrm{m}}} / M_{\mathrm{ISM}}$. In Scoville et al. (2014), three samples were developed: (1) 12 local star forming and starbursting galaxies with global SCUBA and ISM measures; (2) extensive Galactic observations from the Planck Collaboration; and (3) a sample of 28 submillimeter galaxies (SMGs) at $z<3$ having $\mathrm{CO}(1-0)$ measurements. The three samples yielded $\alpha_{850 \mu \mathrm{m}}=1.0,0.79$, and $1.01 \times 10^{20} \mathrm{erg} \mathrm{sec}^{-1} \mathrm{~Hz}^{-1} M_{\odot}{ }^{-1}$, respectively (Scoville et al. 2014). The Planck measurements are particularly convincing - they are of high $\mathrm{S} / \mathrm{N}$, span a large wavelength range and they probe the diversity of Galactic ISM including both $\mathrm{H}_{\mathrm{I}}$ and $\mathrm{H}_{2}$ dominated clouds. The Planck measurements exhibit little variation in their empirical coefficient $\alpha_{250 \mu \mathrm{m}}$, which is equivalent to our $\alpha_{850 \mu \mathrm{m}}$ (Planck Collaboration 2011b). The Planck measurements also determine very well the long wavelength dust emissivity index: $\beta=1.8 \pm 0.1$ (Planck Collaboration 2011a). Lastly, we note that Draine et al. (2007) see no variation in the dust abundance down to $\sim 20 \%$ of solar metallicity and it is reassuring that the low redshift galaxy sample and the high $z$ SMGs yield very similar $\alpha_{850 \mu \mathrm{m}}$.

Taking account of the frequency shifting of the rest frame IR spectral energy distribution (SED) as a function of redshift $(z)$, and assuming one is observing the optically thin RJ tail with opacity index $\beta=1.8$, Scoville et al. (2014) show that the expected continuum flux is

$$
S_{v}=1.15 \frac{M_{\text {ISM }}}{10^{10} M_{\odot}} \frac{(1+z)^{4.8} T_{25} \nu_{350}^{3.8} \Gamma_{R J}}{d_{G p c}^{2}} \mathrm{mJy}
$$

and the implied mass is therefore

$$
M_{\mathrm{ISM}}=\frac{0.868 \times S_{\nu}(m J y) d_{G p c}^{2}}{(1+z)^{4.8} T_{25} \nu_{350}^{3.8} \Gamma_{R J}} 10^{10} M_{\odot},
$$

where the observed frequency is normalized to $350 \mathrm{GHz}$, the dust temperature is normalized to $25 \mathrm{~K}$ and the luminosity distance to $1 \mathrm{Gpc}$.

$\Gamma_{R J}$ is the correction factor for departure from the RJ $v^{2}$ dependence given by:

$$
\begin{aligned}
\Gamma_{R J}(T, v, z)= & 0.672 \times \frac{v_{350}(1+z)}{T_{25}} \\
& \times \frac{1}{e^{0.672 \times v_{350}(1+z) / T_{25}}-1} .
\end{aligned}
$$

In the case of ULIRGs, there are two cautions with respect to application of Equation (4): (1) the observed wavelength must be sufficiently long that the dust emission is optically thin and (2) the mean dust temperature (mass-weighted) for the ISM within the compact nuclear region is likely to be elevated well above the typical global average of $\sim 25 \mathrm{~K}$ for ISM in normal galaxy disks. Modeling the IR SED of Arp 220, Wilson et al. (2014) found that the dust optical depth was $\tau \sim 1.7$ and 5.3 at $678 \mathrm{GHz}$ and $T_{d}=80$ and $197 \mathrm{~K}$ for the east and west nuclei, respectively. Similarly, Matsushita et al. (2009) used earlier SMA observations at $690 \mathrm{GHz}$ to conclude that the dust emission had $\tau \sim 1$ at $450 \mu \mathrm{m}$ and $T_{d}=49$ and $97 \mathrm{~K}$ for the east and west nuclei.

One could correct for modest optical depths by including a factor of $f(\tau)=\left(1-e^{-\tau}\right) / \tau$ in Equation (4) but a more reliable mass estimate is obtained using the lower frequency continuum measurements where the opacity is much less. For the Arp 220 nuclei we therefore use the $350 \mathrm{GHz}$ fluxes and adopt $T_{d}=100 \mathrm{~K}$. This is recognized as an approximation since the two nuclei are different and undoubtedly have temperature gradients. A good derivation of the dust temperature from the observations would require multiple spectral bands, observed at the same resolution and with resolution sufficient to resolve likely temperature gradients.

The adopted dust temperature is consistent with the luminosity outputs of the nuclei. The Arp 220 nuclei have $L_{\text {FIR }} \simeq$ $10^{12} L_{\odot}$; the blackbody radius required to emit this luminosity is $70 \mathrm{pc}$ if the temperature is $100 \mathrm{~K}$. This radius is comparable with the measured sizes of the dust emission peaks, implying that the dust must be heated to $\gtrsim 100 \mathrm{~K}$ throughout this volume in order to emit the observed luminosity. In NGC 6240, the submillimeter flux is much lower and the emission region is much larger; we therefore assume that the continuum is optically thin at $693 \mathrm{GHz}$ and adopt the more typical mean $T_{d} \sim 25 \mathrm{~K}$, since the dust is more spatially extended and the IR luminosity is 3-4 times lower.

Using these values in Equation (4), we find ISM mass estimates for the nuclei in Arp $220(z=0.018)$ and NGC 6240 $(z=0.024)$ in the range $1.6-6.0 \times 10^{9} M_{\odot}$ (see Table 4$)$. Given the uncertainties in the adopted mass-weighted dust temperature we expect these estimates to be uncertain by a factor of at most two for the nuclear sources.

The mass estimates derived from the dust continuum are similar to those obtained from the CO (1-0 and 2-1) emission lines. The total for both nuclei of Arp 220 is $\sim 5 \times 10^{9} M_{\odot}$ (Scoville et al. 1997); the individual east and west nuclei are $\sim 2-3 \times 10^{9} M_{\odot}$ (Downes \& Solomon 1998) and for NGC $62403 \times 10^{9} M_{\odot}$ (Tacconi et al. 1999). The RJ dust continuum obviously avoids the uncertainties in the conversion factor (and the uncertain relative brightness of high $J \mathrm{CO}$ transitions). One issue that could arise in very dense ISM environments is grain growth by coagulation or grain destruction by collisions; these would change the long wavelength dust opacity coefficient in different directions. We can hope that future ALMA submillimeter imaging at higher and matched resolutions at different wavelengths might demonstrate if the opacity spectral index is non-standard.

Combining the masses derived from the RJ dust emission with the source radius determined from the deconvolved dust emission distributions (Table 4), we obtain mean column densities of $\left\langle N_{H 2}\right\rangle \gtrsim 6,15$, and $0.7 \times 10^{24} \mathrm{~cm}^{-2}$ for Arp 220 east and west and NGC 6240, assuming a uniform, spherical distribution of ISM (see Table 4). Estimates of $\left\langle N_{H 2}\right\rangle \sim 4 \times 10^{25} \mathrm{~cm}^{-2}$ were obtained by Sakamoto et al. (2008) for the Arp 220 nuclei, adopting $\tau_{850 \mu \mathrm{m}} \sim 1$ and using the Hildebrand (1983) Galactic dust opacity law. These are lower limits for the column density along the line of sight to the nuclei since they have assumed a 

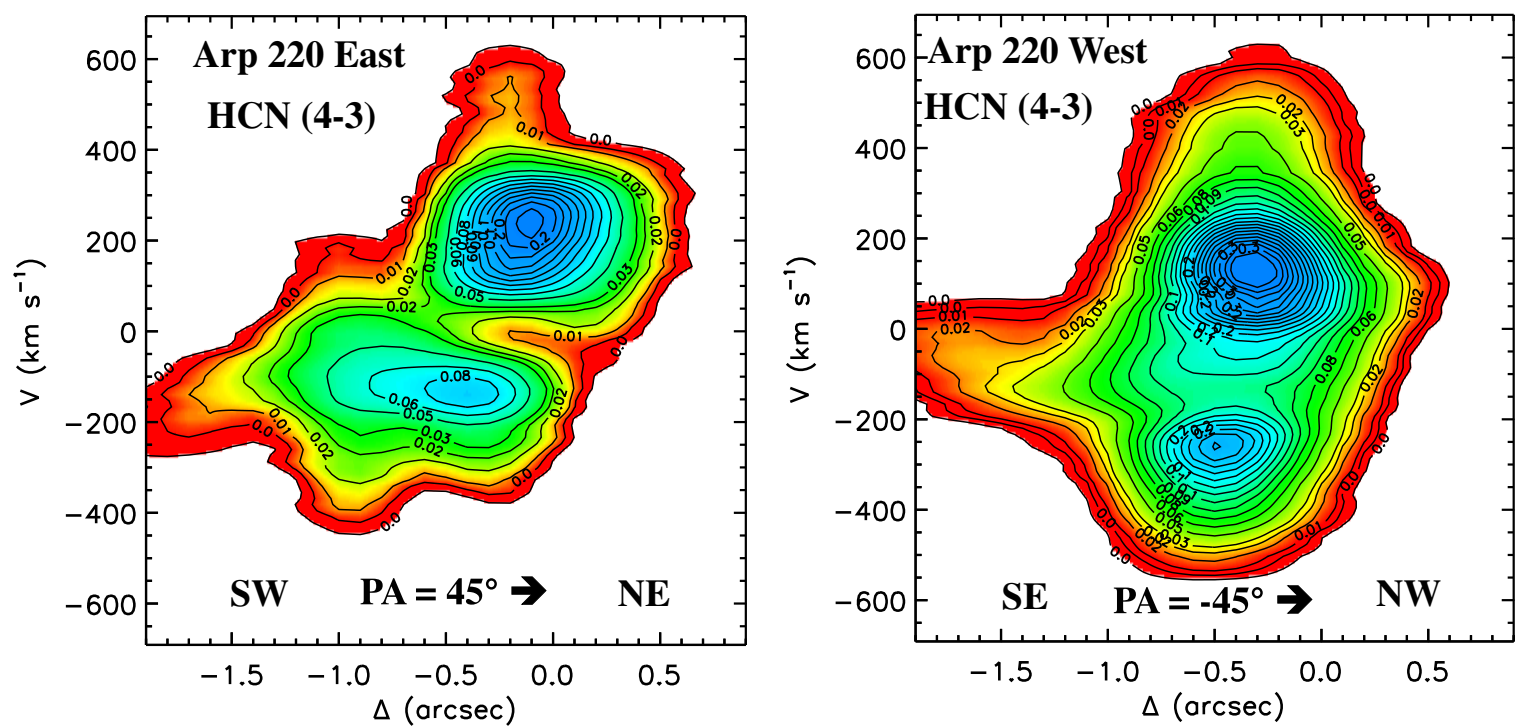

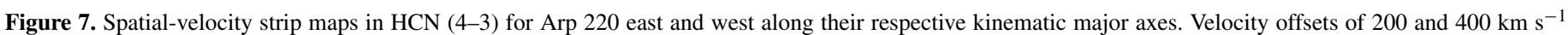
were subtracted at the systemic redshift $z=0.01813$. Contours are at -2 (dashed), 2, 3, 4, 6, 8, 10, 15, 20, 25, . × $\sigma$ where $\sigma=3$ mJy beam ${ }^{-1}$.

Table 4

ISM Masses from Dust Continuum

\begin{tabular}{|c|c|c|c|c|c|c|c|c|c|}
\hline Source & $\begin{array}{c}v_{\mathrm{obs}} \\
(\mathrm{GHz})\end{array}$ & $\begin{array}{l}\text { Flux } \\
(\mathrm{mJy})\end{array}$ & $\begin{array}{l}T_{d}{ }^{\mathrm{a}} \\
(\mathrm{K})\end{array}$ & $\Gamma_{R J}{ }^{\mathrm{d}}$ & $\begin{array}{c}\text { Mass } \\
\left(10^{9} M_{\odot}\right)\end{array}$ & $\begin{array}{c}\text { Diameter } \\
\left({ }^{\prime \prime}\right)\end{array}$ & $\begin{array}{l}\text { Radius } \\
\text { (pc) }\end{array}$ & $\begin{array}{c}\left\langle\Sigma_{\mathrm{gas}}\right\rangle^{\mathrm{c}} \\
\left(M_{\odot} \mathrm{pc}^{-2}\right)\end{array}$ & $\begin{array}{l}\left\langle N_{H 2}\right\rangle^{\mathrm{c}} \\
\left(\mathrm{cm}^{-2}\right)\end{array}$ \\
\hline Arp 220 total & 347.6 & 490 & 100 & 0.917 & 5.9 & & & & \\
\hline Arp 220 east & 347.6 & 161 & 100 & 0.917 & 1.9 & $\lesssim 0.38$ & $\lesssim 69$ & $\gtrsim 1.3 \times 10^{5}$ & $\gtrsim 6.0 \times 10^{24}$ \\
\hline Arp 220 west & 347.6 & 342 & 100 & 0.917 & 4.2 & $\lesssim 0.36$ & $\lesssim 65$ & $\gtrsim 3.1 \times 10^{5}$ & $\gtrsim 1.5 \times 10^{25}$ \\
\hline NGC 6240 & 693.5 & 126 & 25 & 0.468 & 1.6 & 〜 0.8 & $\widetilde{190}$ & $\approx 1.4 \times 10^{4}$ & $\stackrel{7}{7} .0 \times 10^{23}$ \\
\hline
\end{tabular}

Notes. ISM masses derived from the RJ continuum flux using Equation (4) with distances of 74 and 103 Mpc for Arp 220 and NGC 6240.

a Adopted dust temperature used for mass calculation as discussed in the text.

b Angular diameter estimate from deconvolved major axis of dust emission Gaussian fit (Table 3) using the Band 7 measurements for Arp 220 (since the Band 9 dust emission is optically thick) and Band 9 for NGC 6240 (since the S/N is better in Band 9).

${ }^{\mathrm{c}}$ Mean gas surface density and $\mathrm{H}_{2}$ column density assuming the gas mass is distributed uniformly over a disk with the radius given in Column 8 .

d Rayleigh-Jeans correction factor from Equation (4).

uniform distribution of gas and it is more likely that the ISM is centrally peaked. The column density estimates obtained here are more constraining than those by Sakamoto et al. (2008) due to the more up-to-date empirical value for the ISM mass to dust opacity coefficient. Since a gas column of $10^{25} \mathrm{~cm}^{-2}$ would heavily absorb the soft X-rays, these column densities would obscure any X-ray emission from a central AGN in Arp 220 if such exists (Paggi et al. 2013). Thus the non-detection of X-rays does not imply there is no AGN.

\section{NUCLEAR DISK MODELS}

In Arp 220, the molecular gas and dust is likely in disk-like structures, centered on each nucleus of the merging galaxies, separated by only $1^{\prime \prime}$ or a projected separation of $361 \mathrm{pc}$. Although the direct imaging does not conclusively show the large ratio of major/minor axes expected for an edge-on disk, the monotonic velocity gradients along the major axes of both structures (see Figure 7) are suggestive of rotating disks (as seen here and earlier by Sakamoto et al. 1999, 2009). Disk-like configurations for the nuclear gas concentrations in merging systems is also expected theoretically (Barnes \& Hernquist 1996), given the highly dissipative nature of the dense molecular gas damps motion along the rotation axis. Our conclusion that the gas constitutes a significant fraction of the total mass in the nuclei is also to be expected in the late stages of merging since the gas, being more dissipative than the pre-existing stellar systems, will sink to smaller radii more quickly and hence lead to a large gas mass fraction in the nuclei. In NGC 6240, which is probably somewhat less advanced in the merging process, the gas structure peaks near but not on the southern nucleus with a tidal bridge extending toward the northern nucleus. In NGC 6240, the observed kinematics are not ordered with a gradient along a single major axis.

In the Appendix, we describe a kinematic deconvolution technique originally developed by Scoville et al. (1983), which yields a maximum likelihood fit of a simple disk model to the observed HCN line profiles mapped over the area of the disk. The fit obtains a simultaneous solution for a parametric rotation curve and an axisymmetric, radial emissitivity distribution which best fits the observed line profiles seen in HCN (4-3) across Arp 220 east and west and NGC 6240. The objective of this modeling exercise is to yield a self-consistent rotation curve and emissivity distribution that agrees with the observed line profiles, and to see if this dynamic model yields a total dynamical mass consistent with the ISM masses estimated from the dust emission.

The parameters to be solved for are: the velocity dispersion $\sigma_{v}$ of the gas; the inclination $i$ of the disk; the P.A. of the major axis of the disk and the radial emissivity function. The radial 

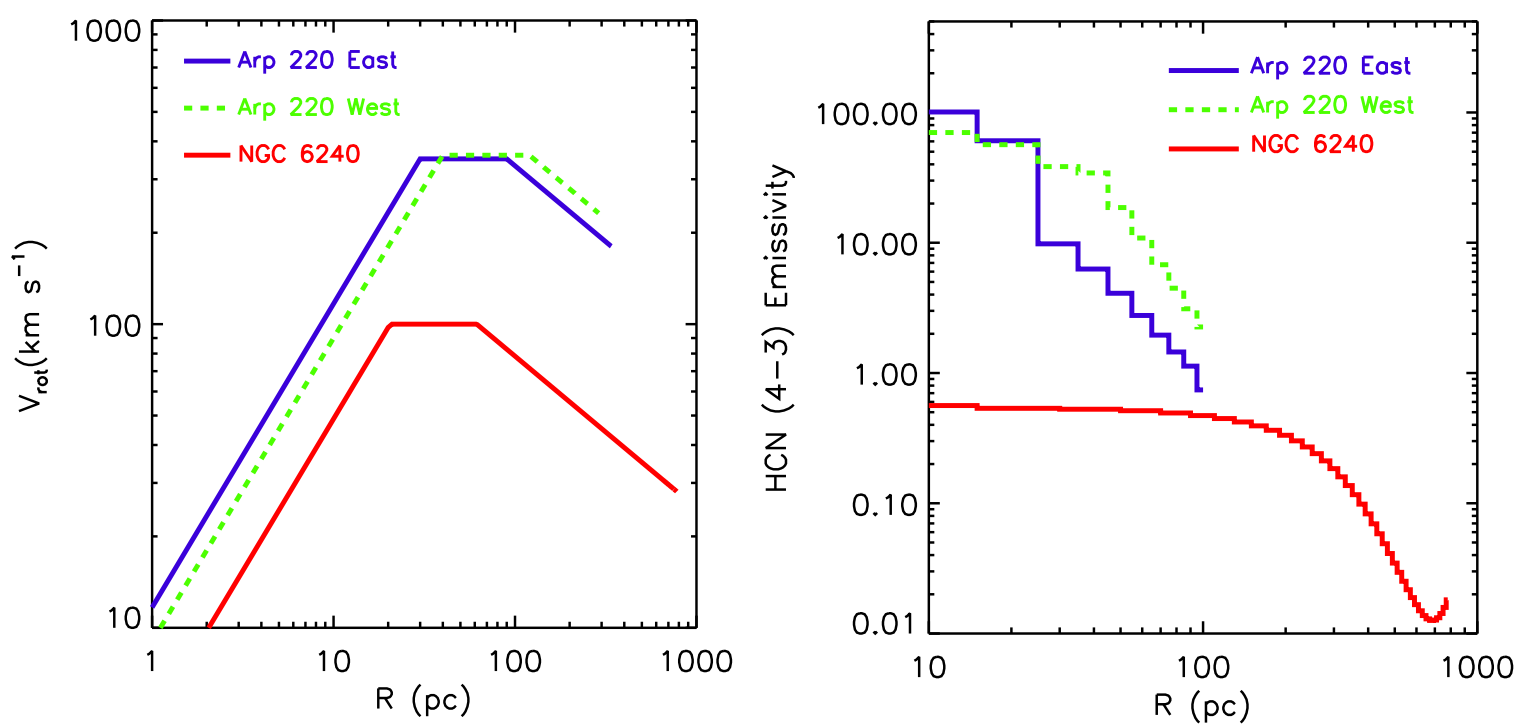

Figure 8. Rotation velocity (left) and emissivity radial distributions (right) derived from the maximum likelihood kinematic deconvolution for the nuclear disks in Arp 220 east and west and NGC 6240.

Table 5

Disk Models

\begin{tabular}{lccccc}
\hline \hline Source & $\begin{array}{c}V_{0} \\
\left(\mathrm{~km} \mathrm{~s}^{-1}\right)\end{array}$ & $\begin{array}{c}R_{0} \\
(\mathrm{pc})\end{array}$ & $\begin{array}{c}\text { Incl. } \\
\left({ }^{\circ}\right)\end{array}$ & $\begin{array}{c}\text { P.A. } \\
\left({ }^{\circ}\right)\end{array}$ & $\begin{array}{c}\sigma_{v} \\
\left(\mathrm{~km} \mathrm{~s}^{-1}\right)\end{array}$ \\
\hline Arp 220 east & 350 & 30 & 71 & 47 & 90 \\
Arp 220 west & 360 & 40 & 64 & -15 & 90 \\
NGC 6240 & 100 & 20 & 70 & -6 & 160 \\
\hline
\end{tabular}

emissivity function was taken to be a step function in radius $(R)$ with 30 equal width bins in $R$ with no imposed continuity between adjacent radial bins. Positions and velocities were measured relative to the spatial and velocity centroids of the $\mathrm{HCN}$ emission; specifically, the centroid position was taken to be $R=0$ for the nucleus and the centroid velocity was adopted as the systemic velocity of each disk. These spatial offsets were in all cases $\leqslant 0$ ' 05 from the dust continuum peaks and the velocity offsets were $\leqslant 20 \mathrm{~km} \mathrm{~s}^{-1}$ from the systemic velocities.

The minimized $\chi^{2}$ parameters are given in Table 5 and the rotation curves and radial emissivity distributions are shown in Figure 8. In both Arp 220 east and west, the general agreement between the model and observed spectra is acceptable given the simplified assumptions of the model-axisymmetry and no radiative transfer considerations. In both Arp 220 nuclei, the structures are consistent with being rotation dominated.

In NGC 6240 the line profiles fits are also good but here the dynamics are dispersion-dominated and the disk structure is therefore not as well constrained. Medling et al. (2011) constrained the black hole mass is the southern nucleus of NGC 6240 using NIR AO measurements of the stellar kinematics using the $\mathrm{CO}$ bandheads and $2.3 \mu \mathrm{m}$. Their range of acceptable masses is $0.9-2 \times 10^{9} M_{\odot}$. This mass is significantly above that indicated by the rotation curve shown in Figure 9, left. The analytic rotation curve used in our fitting did not include a term for a point mass but even if it had included a Keplerian falloff at small radii, the HCN emission is not very centrally concentrated; the modeling cannot therefore constrain the rotation curve there.

The derived emissivity distributions in both nuclei of Arp 220 are strongly concentrated to very small radii, $R \lesssim 20$ pc; in NGC 6240 the emission apparently arises fairly uniformly out to radii $\sim 250 \mathrm{pc}$. For Arp 220 the instrumental resolution (half-power beam width) of the $\mathrm{HCN}$ (4-3) observation is $\sim 0$ '.5 or a diameter of $180 \mathrm{pc}$. Thus the kinematic deconvolution is revealing a source $\sim 5$ times smaller and it will be interesting to see the results of higher resolution ALMA imaging in future cycles.

The kinematics derived from the modeling can be used to estimate dynamical masses for these compact gas concentrations. For the combination of circular orbital motions and uniform random motions, an approximate dynamical mass is obtained from

$$
M(R)=2.3 \times 10^{8}\left(V_{r o t 100}^{2}+\sigma_{v 100}^{2}\right) R_{100} M_{\odot} .
$$

The velocities are normalized to $100 \mathrm{~km} \mathrm{~s}^{-1}$ and the radius to $100 \mathrm{pc}$. For both nuclei in Arp 220 the dynamical masses are $\sim 7 \times 10^{8} M_{\odot}$ at $R=20 \mathrm{pc}$ and $\sim 3 \times 10^{9} M_{\odot}$ at $R=100 \mathrm{pc}$. For NGC 6240, the dynamical mass estimates are $\sim 6 \times 10^{8}$ and $1.4 \times 10^{9} M_{\odot}$ at $R=250$ and $600 \mathrm{pc}$. The vertical structure of the disks is a Gaussian function with thickness (FWHM) given by

$$
\Delta z_{\mathrm{FWHM}}(R) \simeq 100 \sigma_{v 100}\left(\frac{R_{100}}{V_{\text {rot } 100}}\right) \mathrm{pc} .
$$

A more precise formulation of the disk thickness is not warranted at this time since it would require knowledge of the relative fractions of the mass in stars and gas and a more accurate rotation curve. In the event that the disk is entirely self-gravitating and the gas mass is completely dominant then it can be shown that the thickness depends quadratically on the velocity dispersion and is given by

$$
\begin{aligned}
\Delta z_{\mathrm{FWHM}}(R) & \simeq \frac{0.233 \sigma_{v}^{2}}{G \Sigma} \\
& =\frac{53 \sigma_{v 100}^{2}}{\Sigma_{10^{4}}} \mathrm{pc},
\end{aligned}
$$

where $\Sigma$ is the local mass surface density of the disk, here normalized to $10^{4} M_{\odot} \mathrm{pc}^{-2}$ (see Spitzer 1942; Scoville et al. 1997).

Figure 9 shows the dynamical mass, disk thicknesses (from both Equations (6) and (7)) and mean disk density as functions 

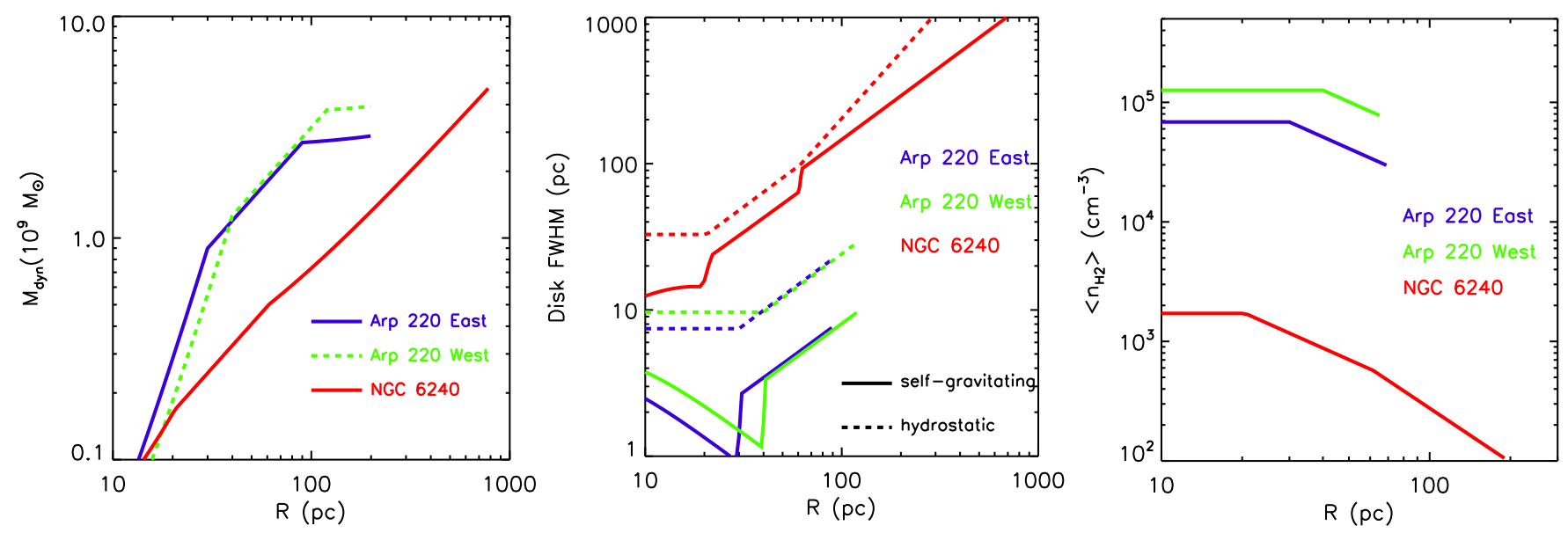

Figure 9. Dynamical mass (Equation (5), left), disk thickness (middle, Equations (6) and (7)) and mean disk gas densities (right) are shown, based on the rotation velocity and velocity dispersions derived from the maximum likelihood kinematic deconvolution for the nuclear disks in Arp 220 east and west and NGC 6240. The mean disk gas densities are shown for the ISM masses from Table 4, distributed with constant mass surface density out to the radius given in Table 4. The scale height as a function of radius is that of the hydrostatic case (solid lines in Figure 9, obtained using Equation (6)). The mass surface density was divided by a factor of 1.36 to remove the He mass contribution.

of radius using the rotation curves from Figure 8 . The density is calculated from the dynamical mass as a function of radius and expressed in terms of equivalent volume density of $\mathrm{H}_{2}$ (in anticipation of the possibility that the gas makes up a large fraction of the overall mass).

\section{HIGH EXCITATION GAS TRACED IN HCN AND CS}

In the Arp 220 nuclei, both the HCN (4-3) and CS (7-6) emission lines are optically thick but subthermally excited. ${ }^{16}$ Their peak brightness temperatures (Table 2) are 20-40 K $(\mathrm{HCN})$ and 7-10 K (CS), implying that they are excited well above the $2.7 \mathrm{~K}$ cosmic microwave background, yet they are significantly less than the $\mathrm{CO}$ brightness temperatures and the $\sim 100 \mathrm{~K}$ dust temperatures for the resolutions probed here. In this section we provide an analytic treatment of the molecular excitation to constrain the physical conditions in the line emission regions.

In the sub-thermal, optically thick regime of excitation, the molecular emission is provided by two processes: collisional excitation by collisions with $\mathrm{H}_{2}$ and line photon radiative trapping (Scoville \& Solomon 1974; Goldreich \& Kwan 1974). Here, we extend the two-level analytic treatment in Scoville \& Solomon (1974) for our analysis of the HCN (4-3) emission in Arp 220. Although one could perform a full multi-level statistical equilibrium calculation for the level populations, this analytic treatment provides better physical insight and yields the dependences on the key parameters: $n_{H_{2}}$ (the $\mathrm{H}_{2}$ volume density), $X_{m}=n_{m} / n_{H_{2}}$ (the molecular abundance relative to $\mathrm{H}_{2}$ ) and $T_{k}$ (the gas temperature). (A similar analytic approach is given by Goldsmith et al. (2012) for the [CII] fine structure line.)

\subsection{Two Level Excitation with Photon Trapping}

We consider a two-level molecular system (e.g., HCN $J=$ 3 and 4) in which the level populations are determined by a balance of collisions with $\mathrm{H}_{2}$, spontaneous decay and line

\footnotetext{
${ }^{16}$ We are not aware of ${ }^{13} \mathrm{C}$ isotope detections for $\mathrm{HCN}$ or CS in Arp 220 but in virtually all Galactic sources with significant $\mathrm{HCN}$ and $\mathrm{CS}$ emission, the lines are optically thick since the rare isotope molecular emissions are much stronger, relative to the abundant isotope, than the isotopic abundance ratios.
}

photon absorption, and stimulated emission with $\tau>1$. The ratio of upper to lower level populations is then given by

$$
\frac{n_{u}}{n_{l}}=\frac{n_{H_{2}}\langle\sigma v\rangle_{u l} g_{u l} e^{-h v / k T_{k}}}{n_{H_{2}}\langle\sigma v\rangle_{u l}+A_{u l} / \tau} .
$$

$g_{u l}$ is the ratio of statistical weights $\left(=g_{u} / g_{l}\right),\langle\sigma v\rangle_{u l}$ is the downward collision rate coefficient and $T_{k}$ is the gas kinetic temperature. In Equation (8), the spontaneous decay rate $A_{u l}$ has been reduced by the photon escape probability, $\beta=\left(1-e^{-\tau}\right) / \tau \simeq 1 / \tau$ for $\tau>1$. The excitation temperature $\left(T_{u l}\right)$ characterizing the level populations is then

$$
\begin{gathered}
\frac{T_{u l}}{T_{k}}=\frac{1}{1+\frac{T_{k}}{T_{0}} \ln (1+\chi)}, \\
\text { where } \chi=\frac{A_{u l} / \tau}{n_{H_{2}}\langle\sigma v\rangle_{u l}}
\end{gathered}
$$

and $T_{0}=h v / k$. The line optical depth is given by

$$
\tau=A_{u l} \frac{g_{u l} c^{3}}{8 \pi v^{3} d v / d r} n_{l}\left(1-e^{-T_{0} / T_{u l}}\right) .
$$

Here, $d v / d r$ is the line-of-sight velocity gradient.

Combining Equations (10) and (11), one sees that the excitation temperature in an optically thick transition is in fact independent of the spontaneous decay rate $\left(A_{u l}\right)$. This is because transitions with higher spontaneous decay rates also have proportionally higher optical depth and hence lower photon escape probabilities. Although this has been shown before (Scoville \& Solomon 1974; Goldreich \& Kwan 1974), we restate this result here since it is often asserted that the greater dipole moment molecules have a higher critical density due to their more rapid spontaneous decay; this is not physically correct for optically thick transitions such as those of HCN and CS. In fact, the excitation temperature is independent of the $A$-coefficient; hence, the line brightness temperature depends only on the factor $n_{H_{2}} n_{m} /(d v / d r)$ where $n_{m}$ is the volume density of the molecules (reflected above in $n_{l}$ ).

To proceed further, we make the approximation that the rotational level populations can be characterized by a single 
excitation temperature $T_{x}$. This excitation temperature can then be used to relate the lower level population $n_{l}$ ) to the total molecular density $\left(n_{m}\right)$ via a Boltzmann distribution. For a linear molecule, the rotational partition function is $Z=k T_{x} /(h B)$ where $B$ is the rotation constant. Thus,

$$
\begin{aligned}
n_{l} & =g_{l} e^{-E_{l} / k T_{x}} n_{m} / Z \\
& =\frac{g_{l} e^{-E_{l} / k T_{x}} n_{m} h B}{k T_{x}} .
\end{aligned}
$$

For linear molecules like HCN and CS, the transition $J \rightarrow J-1$ has $E_{l}=h B\left(J^{2}-J\right)$ and $T_{0}=h v / k=2 J h B / k(B=44.32$ and $24.50 \mathrm{GHz}$ for $\mathrm{HCN}$ and $\mathrm{CS}$ ). Equation (9) then becomes

$$
\frac{T_{x}}{T_{k}}=\frac{1}{1+\frac{T_{k}}{T_{0}} \ln \left(1+\psi f\left(T_{x}\right)\right)}
$$

where $\quad \psi=\frac{64 \pi(J B / c)^{3}}{\left(n_{m} /(d v / d r)\right) n_{H_{2}} g_{u}\langle\sigma v\rangle_{u l}}$

$$
\text { and } f\left(T_{x}\right)=\frac{k T_{x} / h B}{e^{-h B\left(J^{2}-J\right) / k T_{x}}-e^{-h B\left(J^{2}+J\right) / k T_{x}}} .
$$

\subsection{HCN (4-3) Excitation in Arp 220}

This two-level formalism can be applied to the observed $\mathrm{HCN}$ (4-3) emission in Arp 220 to constrain the gas density in the nuclear sources. In the nuclei at radii less than $\sim 65 \mathrm{pc}$ we adopt a gas kinetic temperature $T_{k}=100 \mathrm{~K}$, based on the dust blackbody-limit temperature and the expectation that at densities above $10^{4} \mathrm{~cm}^{-3}$ the gas should be thermally coupled to the dust. This high kinetic temperature is consistent with the brightness temperatures observed for $\mathrm{CO}$ (Sakamoto et al. 2009). For HCN (4-3), the peak line brightness temperatures are $\sim 20$ and $40 \mathrm{~K}$ (Table 3). Assuming the line is optically thick (as in Galactic sources), these brightness temperatures imply excitation temperatures $T_{x} \sim 20$ and $40 \mathrm{~K}$. In the evaluation below, we adopt $T_{x} \simeq 40 \mathrm{~K}$ and therefore the gas density will be constrained to that giving $T_{x} / T_{k} \simeq 0.4$ in Equation (13).

For $\mathrm{HCN}, h B / k=2.13 \mathrm{~K}$ and for the $J=4-3$ transition, $T_{0}=17.0 \mathrm{~K}$ with $A_{4-3}=2.1 \times 10^{-3} \mathrm{~s}^{-1}$. The collision rate coefficients for $\mathrm{HCN} J=4$ were taken from the $\mathrm{He}-\mathrm{HCN}$ rate coefficients calculated by Dumouchel et al. (2010), scaled by a factor of 1.36 to go to $\mathrm{HCN}-\mathrm{H}_{2}$ (see Green \& Thaddeus 1976). For this analytic analysis, it is appropriate to use the sum of the collision rate coefficients out of the $J=4$ upper level to any other rotational level (both below and above $J=$ 4) since all of these transitions couple the level to the gas kinetic temperature. This summed rate coefficient is $\langle\sigma v\rangle_{J=4}=$ $2.68 \times 10^{-10}\left(T_{k} / 100\right)^{-0.22}$ for $T_{k} \simeq 50-150 \mathrm{~K}$ (using the tables given in Schöier et al. 2005).

For the characteristic line-of-sight velocity gradient, we divide the line FHWM $\left(\sim 500 \mathrm{~km} \mathrm{~s}^{-1}\right)$ by the diameter of the nuclear sources (130 pc, Table 4), yielding $d v / d r \sim$ $4 \mathrm{~km} \mathrm{~s}^{-1} \mathrm{pc}^{-1}\left(=1.3 \times 10^{-13} \mathrm{sec}^{-1}\right)$. Lastly, we set the abundance of $\mathrm{HCN}$ relative to $\mathrm{H}_{2}$ to the typical value obtained for Galactic sources $\left(X_{\mathrm{HCN}} \sim 2 \times 10^{-8}\right.$; Bergin et al. 1996; Lahuis \& van Dishoeck 2000) so that the molecular volume density is $n_{m}=X_{\mathrm{HCN}} n_{H 2}$.

\subsection{CS (7-6) Excitation in Arp 220}

For CS (7-6), the peak line brightness temperatures are $\sim 7.5$ and $10 \mathrm{~K}$ (Table 3), i.e., about one fourth of those for HCN (4-3), implying that $T_{x} / T_{k} \simeq 0.1$ in Equation (13). For CS, $h B / k=1.175 \mathrm{~K}$ and for the $J=7-6$ transition, $T_{0}=16.5 \mathrm{~K}$ with $A_{7-6}=8.4 \times 10^{-4} \mathrm{sec}^{-1}$. The collision rate coefficients for CS (Schöier et al. 2005) have total $\langle\sigma v\rangle_{J=7}=2.87 \times$ $10^{-10}\left(T_{k} / 100\right)^{0.15}$, once again summing all rate coefficients from $J=7$. We use the same line-of-sight velocity gradient as for $\mathrm{HCN}$, i.e., $d v / d r=1.3 \times 10^{-13} \mathrm{sec}^{-1}$. For CS, the Galactic and nearby galaxies abundance is typically $X_{C S} \sim 5 \times 10^{-9}$ (Paglione et al. 1995; Shirley et al. 2003; Bergin et al. 1997).

\subsection{Densities Required for the Observed Emission}

Figure 10 (left) shows the resulting ratios of $T_{x} / T_{k}$ obtained for HCN (4-3) and CS (7-6) from Equation (13) as a function of density. The observed fiducial values for $T_{x} / T_{k}$ are $\sim 0.4$ and 0.1 in Arp 220 west for HCN and CS, respectively - these are shown by the horizontal bars. For the adopted molecular abundances the required densities are $n_{H_{2}}=2.0$ and $3.0 \times 10^{5} \mathrm{~cm}^{-3}$ for $\mathrm{HCN}$ and CS. The densities derived from this analytic two-level treatment are within $10 \%$ of those derived using a full multilevel code such as RADEX. Figure 10 (right) shows the line optical depths as a function of density, indicating that both lines are optically thick ( $\tau=130$ and 3$)$ - the HCN line optical depth is over 100 !

It is noteworthy that these two lines indicate fairly consistent gas volume densities $\left(2.0\right.$ and $\left.3.0 \times 10^{5} \mathrm{~cm}^{-3}\right)$ without requiring abnormal molecular abundances, so we conclude that $n_{H 2} \sim$ $2 \times 10^{5} \mathrm{~cm}^{-3}$. This volume density for the line emitting gas is only slightly higher than the mean volume density $\left(\sim 10^{5} \mathrm{~cm}^{-3}\right.$, estimated from the dynamical mass and from the dust-based mass estimate). The fact that these agree so closely implies the volume is almost entirely filled with gas at density $1-2 \times 10^{5} \mathrm{~cm}^{-3}$. Thus, the nuclear gas configuration must be quite uniform and not a cloudy or swiss cheese-like structure.

The above analysis considers only collisional excitation. In some instances, the excited rotational levels can be populated via absorption of near/mid infrared dust continuum photons in the molecular vibrational bands, followed by spontaneous decay to the excited rotational levels of the ground vibrational state (Carroll \& Goldsmith 1981). For CS and HCN, this mode starts for dust blackbody temperatures above $\sim 120$ and 400, respectively, for $\mathrm{CS}$ and $\mathrm{HCN}$ but considerably higher $T_{d}$ are required for the high $J$ states observed here (see Carroll \& Goldsmith 1981).

\section{DISCUSSION}

Here, we briefly discuss the constraints on variations in the $\mathrm{CO}$ conversion factor used to translate CO (1-0) emission line flux to mass of $\mathrm{H}_{2}$ and the estimation of SFRs from the IR fine structure lines such as CII.

\section{1. $\mathrm{CO}$-to- $\mathrm{H}_{2}$ Conversion Factor}

Using the masses derived from the dust emission in Section 4 and Table 4, we can assess the effective CO (1-0) conversion factor for the Arp 220 nuclei (assuming the masses obtained from the dust emission are valid). To circumvent the problem that angular resolution of existing $\mathrm{CO}(1-0)$ is insufficient to separate the two nuclei, and to separate their emission from the more extended $\mathrm{CO}$ emission, we make use of the $\mathrm{CO}(2-1)$ 

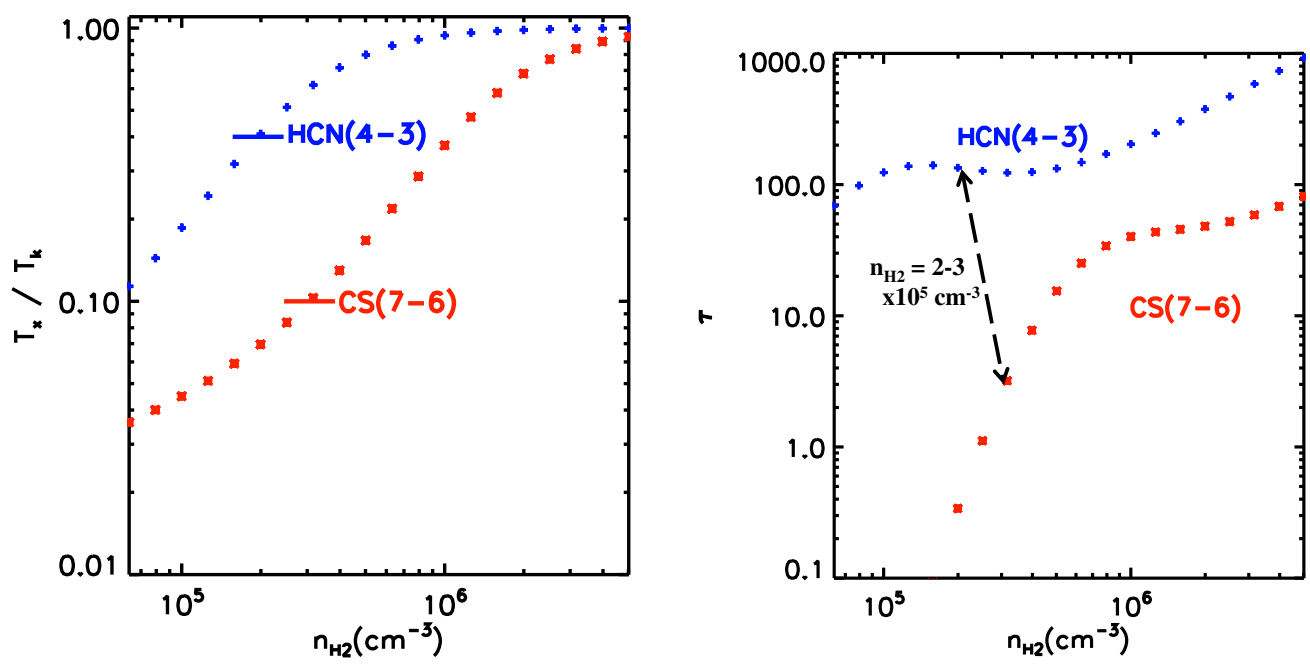

Figure 10. Left: the ratio of the level excitation temperature to the gas kinetic temperature $\left(T_{x} / T_{k}\right)$ is shown as a function of density for the HCN (4-3) and CS (7-6) lines, calculated from Equation (13) for the parameters discussed in the text, specifically $T_{k}=100 \mathrm{~K}$ and standard galactic abundances $2 \times 10^{-8}$ and $2 \times 10^{-9}$ for $\mathrm{HCN}$ and CS relative to $\mathrm{H}_{2}$. The observed thermalization ratios (derived from the line brightness temperatures, see the text) are $\sim 0.4$ and 0.1 , requiring $n_{\mathrm{H}_{2}}=2.0$ and $3.0 \times 10^{5} \mathrm{~cm}^{-3}$, respectively, for HCN and CS. Right: line optical depths are shown. The optical depths corresponding to the solution densities are $\sim 130$ and 3 for the HCN (4-3) and CS (7-6) lines, averaged over the line profile.

Table 6

Arp 220: CO and Dust Masses

\begin{tabular}{lcccc}
\hline \hline & $S_{2-1} \Delta V$ & $S_{1-0} \Delta V$ & $\begin{array}{c}\text { ISM(CO) } \\
10^{9} M_{\odot}\end{array}$ & $\begin{array}{c}\text { ISM(dust) } \\
10^{9} M_{\odot}\end{array}$ \\
\hline East & 120 & $30-46$ & $3.9-6.0$ & 2.0 \\
West & 187 & $47-72$ & $6.0-9.2$ & 4.2 \\
\hline
\end{tabular}

Notes. CO $(2-1)$ fluxes for the nuclei are from Sakamoto et al. (1999). Similar 2-1 fluxes are given by Downes \& Solomon (1998): 130 and $220 \mathrm{Jy} \mathrm{km} \mathrm{s}{ }^{-1}$ (assuming their table entries for the east and west nuclei are reversed). Flux units are $\mathrm{Jy} \mathrm{km} \mathrm{s}^{-1}$. A lower limit on the $1-0$ flux is obtained by assuming the flux scales as $v^{2}$ and the ratio is therefore 4:1. The upper limit is obtained by adopting the global $\mathrm{CO}(2-1) /(1-0)$ flux ratio of Arp $220(1071 / 410=$ 2.6 Scoville et al. 1997). ISM(CO) is the ISM mass estimate obtained from the $\mathrm{CO}(1-0)$ flux assuming the standard Galactic CO-to-H2 conversion factor and ISM(dust) is the ISM masses estimated from dust RJ continuum flux as listed in Table 4.

observations of Sakamoto et al. (1999) at 0'.5 resolution. To estimate the effective (1-0) flux one can scale the (2-1) flux by a factor of one fourth, assuming the two lines are thermalized and have constant brightness temperature. Alternatively, one could scale by the global $(2-1) /(1-0)$ flux ratio $=2.6$ (Scoville et al. 1997). The $1-0$ flux obtained using this latter ratio should be viewed as an upper limit since the global flux ratio includes low excitation $\mathrm{CO}$ from larger galactic radii. These estimates are listed in Table 6.

For the standard Galactic CO-to- $\mathrm{H}_{2}$ conversion factor $\alpha_{C O(1-0)}=3 \times 10^{20} \mathrm{H}_{2} \mathrm{~cm}^{-2}\left(\mathrm{~K} \mathrm{~km} \mathrm{~s}^{-1}\right)^{-1}$, the $\mathrm{H}_{2}$ mass is $\mathrm{M}_{H_{2}}=1.18 \times 10^{4} S_{1-0} \Delta V d_{\mathrm{Mpc}}^{2}$ (see the Appendix in Sanders et al. 1991). The resulting CO-based mass estimates are given in column (4) of Table 6 after multiplying by a factor of 1.36 to account for He. The ISM mass estimates from the CO (1-0) using the standard Galactic $\mathrm{CO}-$ to- $\mathrm{H}_{2}$ conversion factor are two to three times higher than the estimates obtained from the dust continuum (see Table 6). The lower CO estimates are probably more valid for the nuclei since the flux ratio of the high excitation gas is very likely to be thermalized and therefore have the limiting 4:1 ratio.

For self-gravitating molecular clouds or galactic nuclei where the gas is the dominant mass component, one expects the
CO-to- $\mathrm{H}_{2}$ conversion factor to scale as $\sqrt{n_{H_{2}}} / T_{k}$ (Dickman et al. 1986; Bryant \& Scoville 1996), relative to the density and temperature of the Galactic giant molecular clouds (GMCs) for which the standard conversion factor was derived. The Galactic GMC ratio is $\sim \sqrt{300} / 10 \sim 1.7$ and the ratio for the Arp $220 \mathrm{nu}-$ clei is $\sim \sqrt{10^{5}} / 100 \sim 3.2$. Thus a factor of $\sim 2$ change in $\alpha_{C O(1-0)}$ is to be expected; this is consistent with the discrepancy between the CO- and dust-based ISM mass estimates given in Table 6.

Thus we conclude that the effective CO-to- $\mathrm{H}_{2}$ conversion factor in the Arp 220 nuclei is approximately a factor of two reduced from the standard Galactic value. Most of this reduction can be attributed to the expected changes in the mean gas density and gas kinetic temperature. The $\mathrm{CO}-$ to- $\mathrm{H}_{2}$ conversion factor can also be reduced if the line width is increased due to there being a significant stellar mass contribution in the self-gravitating region containing the molecular gas (Bryant \& Scoville 1996; Downes \& Solomon 1998). In the Arp 220 nuclei, we find that this mechanism is not necessitated since the apparent changes in the conversion factor can be largely attributed to the expected scaling for higher $T_{k}$ and density. Lastly, we point out that the masses estimated here from the dust emission and from the $\mathrm{CO}$ (as discussed above) are consistent with the dynamical mass estimated from the kinematic modeling (as shown in Figure 9, left). Thus the derived conversion factor, reduced by a factor of two from the standard one, is certainly not implausible.

\subsection{SFRs from CII and the "IR Line Deficits"}

The $158 \mu \mathrm{m}$ CII line is the most luminous far-infrared line from dusty star forming regions and a number of investigations have attempted to use the CII line fluxes to probe SFRs (see Malhotra et al. 2001; Stacey et al. 2010). However, it is now well established that in many ULIRGs the CII line luminosity is suppressed by factors of 10-100 relative to the far infrared luminosity (Luhman et al. 2003; Graciá-Carpio et al. 2011; Díaz-Santos et al. 2014). The latter is a robust indicator of dust obscured SF in galaxies not having strong AGNs.

There are a number of explanations that have been proposed to account for the suppressed line flux: (1) a sufficiently high density in the emission line regions that the fine structure 
levels are collisionally de-excited (i.e., the gas density is above the fine structure transition critical density), (2) some of the UV luminosity that would normally photoionize the $\mathrm{H}$ II and photodissociation region (PDR) gas is absorbed by dust, thus enhancing $L_{I R}$ relative to $L_{\mathrm{CII}}$; or (3) emission line flux is suppressed by absorption due to overlying dust.

For the CII line, the critical densities are $n_{e} \simeq 50 \mathrm{~cm}^{-3}$ for ionized gas at $10^{4} \mathrm{~K}$ and $n \simeq 3000 \mathrm{~cm}^{-3}$ for neutral gas at $100 \mathrm{~K}$. The mean gas densities estimated above for the Arp 220 nuclei $\left(n_{H_{2}} \simeq 5 \times 10^{5} \mathrm{~cm}^{-3}\right)$ are 100 times higher than the neutral gas critical density; thus in Arp 220, collisional suppression can fully account for the line deficit. In addition, the foreground dust opacities in Arp 220 are such that the dust is optically thick out to $\lambda \gtrsim 300 \mu \mathrm{m}$, so it is expected that there will be substantial dust absorption of the line emission, even if the intrinsic emission luminosity is normal.

Díaz-Santos et al. (2013) find that the CiI line deficit is correlated with there being warm dust in the LIRGs and ULIRGs. They suggest that this is consistent with absorption by dust in the PDRs of the UV longward of the Lyman limit. This is the UV that is responsible for ionizing CII in the PDR regions. Given the physical conditions in Arp 220, the $\mathrm{CII}_{\mathrm{II}}$ line suppression is inevitable due to collisional de-excitaiton and the line emission will also be absorbed by overlying dust so it is not necessary to invoke this additional mechanism. It is important also to recognize that a significant fraction of the $\mathrm{CII}_{\mathrm{II}}$ emission (25\%-50\%) probably arises from the $\mathrm{H}$ II regions (as opposed to PDRs) and this would not be affected by the PDR dust. The $\mathrm{H}$ II region $\mathrm{CII}$ emission component would therefore be at its normal strength relative to the SFR; since this component is a significant fraction of the normal CII emission, it would then be difficult to reach line deficits reaching factors of 10-100.

\subsection{Eddington Limit}

In nuclear SB and AGN sources with a dusty ISM, the absorption of radiation by the dust can produce an outward radiation pressure force comparable or larger than gravity, leading to self-regulation of the activity and radiatively driven outflows (Scoville et al. 2001; Scoville 2003; Thompson et al. 2005; Murray et al. 2005). Thus there exists an Eddington limit on the luminosity-to-mass ratio and above this limiting $\mathrm{L} / \mathrm{M}$ the ISM is driven outward and hence the AGN and SB fueling is cut off.

Given the rotational curve for a galactic nucleus, one can write the Eddington luminosity as

$$
\begin{aligned}
L_{\mathrm{Edd}}= & \frac{4 \pi G M(<R) c}{\kappa_{R}}=\frac{4 \pi V_{\mathrm{rot}}(R)^{2} R c}{\kappa} \\
\simeq & 1.5 \times 10^{12} L_{\odot}\left(\frac{V_{\mathrm{rot}}}{350 \mathrm{~km} \mathrm{~s}^{-1}}\right)^{2}\left(\frac{R}{40 \mathrm{pc}}\right) \\
& \times\left(\frac{10 \mathrm{~cm}^{2} \mathrm{~g}^{-1}}{\kappa_{R}}\right) \text { for Arp220 }
\end{aligned}
$$

and

$$
\begin{aligned}
\simeq & \times 10^{11} L_{\odot}\left(\frac{V_{\text {rot }}}{100 \mathrm{~km} \mathrm{~s}^{-1}}\right)^{2}\left(\frac{R}{100 \mathrm{pc}}\right) \\
& \times\left(\frac{10 \mathrm{~cm}^{2} \mathrm{~g}^{-1}}{\kappa_{R}}\right) \text { for NGC6240, }
\end{aligned}
$$

where we assumed a relatively high value of the Rosseland opacity $\kappa_{R}$ for the dust.
The effective opacity for the dust to absorb the outflowing radiative momentum of course depends on the typical wavelength of the photons at each radius. The original luminosity from the AGN or SB is in the optical-UV, but if the overlying dust is optically thick, these photons are absorbed in the first column with $\mathrm{A}_{V} \sim 1 \mathrm{mag}$ ( or $\mathrm{N}_{H}=2 \times 10^{21} \mathrm{~cm}^{-2}$ for standard gas-to-dust abundance). The absorbed radiation is then re-emitted in the infrared at successively longer wavelengths at larger radii since the dust temperatures decreases at larger radii. The coupling of the dust to the radiation field depends on the effective dust opacity at the characteristic wavelength of the photons at each radius. The Eddington ratio, $\mathrm{L} / \mathrm{M}$ or equivalently $L /\left(V^{2} / R\right)$, will therefore decrease at larger radii in an optically thick dust cloud since the effective opacity is reduced for longer wavelength photons. Semenov et al. (2003) give $\kappa_{R}(T) \simeq 2 \mathrm{~cm}^{2} \mathrm{~g}^{-1}(T / 100 \mathrm{~K})^{2}$ for $T \lesssim 200 \mathrm{~K}$ and $\kappa_{R}(T) \simeq 5 \mathrm{~cm}^{2} \mathrm{~g}^{-1}$ for $T \gtrsim 200 \mathrm{~K}$ for a dustto-gas ratio normalized to the Milky Way value.

Prior treatments of the Eddington limit in the context of SBs have lacked strong constraints on the radial profile of the radiation field to compare with the self-gravitating mass. The $\mathrm{SFR} \simeq 120 M_{\odot} \mathrm{yr}^{-1}$ for the west nucleus of Arp 220 based on the infrared luminosity. We can convert this to a bolometric power by assuming a standard Kroupa initial mass function:

$$
\begin{aligned}
L_{\mathrm{bol}}= & \epsilon \mathrm{SFR} c^{2} \simeq 1.1 \times 10^{12} L_{\odot} \\
& \times\left(\frac{\mathrm{SFR}}{100 M_{\odot} \mathrm{yr}^{-1}}\right) \times\left(\frac{7 \times 10^{-4}}{\epsilon}\right) .
\end{aligned}
$$

For Arp 220, the Eddington ratio is then near-unity for the western nucleus and sub-Eddington for the eastern nucleus by a factor of a few for the nominal $\kappa_{R}$.

Alternatively, one could take the $\mathrm{HCN}$ (4-3) emissivity profiles and assume that $L_{\mathrm{bol}}(R)$ tracks the HCN emissivity: $L_{\text {bol }} \propto L_{\mathrm{HCN}(4-3)}$ (Figure 8) and then take the dynamical mass as a function of radius from Figure 9. The emissivity is rising rapidly at smaller radii in the nuclei of Arp 220, but the enclosed mass must be falling. Thus, the Eddington ratio should be rising toward the core of the nuclei, ultimately leading to an inner radius where the Eddington ratio exceeds unity and material would be ejected. Given the uncertainties in the mass and emissivity distributions derived here; we defer of numerical analysis of this approach to a later data set with higher spatial resolution.

\section{SUMMARY AND PERSPECTIVE}

These ALMA data probing the high excitation gas in the nuclei of two luminous IR galaxies clearly reveal the spectacular gas concentrations and physical conditions in these late stage mergers. Consistent values are obtained for the gas mass derived from the RJ dust emission and for the dynamical mass derived from modeling the observed emission line profiles.

The nuclear disks in Arp 220 are found to be extraordinarily compact with radii $\leqslant 65 \mathrm{pc}$ and masses $\sim 2 \times 10^{9} M_{\odot}$. The typical disk thickness $\sim 10 \mathrm{pc}$ is estimated from the line profile velocity dispersion relative to the rotation velocities. The gas densities derived from the excitation requirements of the HCN and CS emission are consistent, yielding $n_{H_{2}}=2 \times 10^{5} \mathrm{~cm}^{-3}$. Since these densities are within a factor of two of the volumeaverage density for the nuclear gas, this high density gas must uniformly fill the volume and not be cloudy or swiss cheese-like (see Section 6.4).

There has been long standing uncertainty with respect to using the $\mathrm{CO}$ emission from ULIRGs to estimate gas masses 
since the gas may be hotter and denser. These changes affect the CO-to-H2 conversion factor in opposite directions and therefore reduce the shifts in $\alpha_{\mathrm{CO}}$. The millimeter lines can also have enhanced emissivity per unit gas mass if the central potential well of the galaxy nucleus is broadening the line emission. In the future, the effects can be resolved with highresolution ALMA imaging-measuring the CO lines and dust emission as a function of radii to differentiate the different dependences.

Most of the analysis in this paper has focused on Arp 220 - it is brighter and hence the data has higher $\mathrm{S} / \mathrm{N}$ and its structure, consisting of two disks centered on the near infrared nuclei, is simpler. On the other hand, it is fair to ask: why is NGC 6240 less luminous than Arp 220 while its twin AGNs are more active? Is this simply due to the incredibly high column densities $\left(>10^{24-25} \mathrm{~cm}^{-2}\right)$ in Arp 220, which would preclude easy detection of an X-ray AGN there (see Paggi et al. 2013), Or is it the difference in total gas content, orbital configuration and/or mass distribution, gas affecting the AGN activity? The observations and modeling presented here clearly show a three to four times lower gas content in NGC 6240 with three times larger radius (see Table 4). In addition, most of this ISM is associated with the southern nucleus in NGC 6240 as compared with a more equal distribution in Arp 220. Despite the above differences, both systems, with nuclei separated by 361-713 pc (projected), are clearly on a precipitous "death" spiral to coalesence in next $\sim 10-20$ Myrs and subsequent rebirth as a more massive single galaxy.

We thank the referee for a very thorough reading of the manuscript. We thank Zara Scoville for proofreading the manuscript. K.S. is supported by the National Radio Astronomy Observatory, which is a facility of the National Science Foundation operated under cooperative agreement by Associated Universities, Inc. This paper makes use of the following ALMA data: ADS/JAO.ALMA 2011.0.00175.S. ALMA is a partnership of ESO (representing its member states), NSF (USA), and NINS (Japan); together with NRC (Canada) and NSC and ASIAA (Taiwan), in cooperation with the Republic of Chile. The Joint ALMA Observatory is operated by ESO, AUI/NRAO, and NAOJ.

\section{APPENDIX}

\section{KINEMATIC MODELING AND DECONVOLUTION}

We make use of the kinematic deconvolution technique developed by Scoville et al. (1983). This yields a maximum likelihood solution for the disk model with axisymmetric emissivity distribution, rotation curve, and velocity dispersion, which yields line profiles best matching the observed line profiles given the instrumental spatial and kinematic resolutions. The technique is analogous to Doppler radar of rotating planets and in our earlier application to the $\mathrm{CO}$ single dish observations of NGC 1068, it revealed a molecular ring at $\sim 12^{\prime \prime}$ radius although the single dish beam was $60^{\prime \prime}$ resolution (Scoville et al. 1983).

We used the same algorithm described in Scoville et al. (1983), with the addition of an external computation loop, varying rotation curve parameters to minimize the overall $\chi^{2}$ of the observed line profiles compared with those "observed" from the model using the observational resolution parameters. The rotation curve was parameterized by the simple three-part function:

$$
\begin{aligned}
& V(R)=V_{0} \frac{R}{R_{0}} \mathrm{~km} \mathrm{~s}^{-1} \text { at } R \leqslant R_{0} \\
& V(R)=V_{0} \mathrm{~km} \mathrm{~s}^{-1} \text { at } R_{0}<R<3 R_{0} \\
& V(R)=V_{0}\left(\frac{3 R_{0}}{R}\right)^{1 / 2} \mathrm{~km} \mathrm{~s}^{-1} \text { at } R \geqslant 3 R_{0},
\end{aligned}
$$

where $V_{0}$ and $R_{0}$ were parameters varied to minimize the $\chi^{2}$. The exact form of the rotation curve is somewhat arbitrary; however, it is reasonable to have a rising portion $\left(R \leqslant R_{0}\right)$ and then a fairly flat portion to mimic observed galactic rotation curves. The last segment, a Keplerian falloff, is physically motivated by the finite radial extent of the massive nuclear disks.

The remaining parameters are: the velocity dispersion $\sigma_{v}$ of the gas, the inclination $i$ of the disk, the P.A. of the major axis of the disk and the emissivity as a function of radius. The latter was taken to be a step function in radius with 30 equal width bins in $R$ with no imposed continuity between adjacent radial bins. Positions and velocities were all measured relative to the spatial and velocity centroid of the HCN emission; i.e., the centroid position and velocity is adopted as the nucleus $(R=0)$ and the systemic velocity of each disk. (These offsets were in all cases $\leqslant 0$.'05 and $\leqslant 20 \mathrm{~km} \mathrm{~s}^{-1}$ from the dust continuum nuclear peaks and the systemic velocities.)

\section{REFERENCES}

Aalto, S., Wilner, D., Spaans, M., et al. 2009, A\&A, 493, 481

Antón, S., Browne, I. W. A., Marchã, M. J. M., Bondi, M., \& Polatidis, A. 2004, MNRAS, 352, 673

Barcos-Muñoz, L., Leroy, A. K., Evans, A. S., et al. 2014, ApJ, 799, 10

Barnes, J. E., \& Hernquist, L. 1992, ARA\&A, 30, 705

Barnes, J. E., \& Hernquist, L. 1996, ApJ, 471, 115

Bergin, E. A., Goldsmith, P. F., Snell, R. L., \& Langer, W. D. 1997, ApJ, 482, 285

Bergin, E. A., Snell, R. L., \& Goldsmith, P. F. 1996, ApJ, 460, 343

Bryant, P. M., \& Scoville, N. Z. 1996, ApJ, 457, 678

Bryant, P. M., \& Scoville, N. Z. 1999, AJ, 117, 2632

Carroll, T. J., \& Goldsmith, P. F. 1981, ApJ, 245, 891

Díaz-Santos, T., Armus, L., Charmandaris, V., et al. 2013, ApJ, 774, 68

Díaz-Santos, T., Armus, L., Charmandaris, V., et al. 2014, ApJL, 788, L17

Dickman, R. L., Snell, R. L., \& Schloerb, F. P. 1986, ApJ, 309, 326

Downes, D., \& Eckart, A. 2007, A\&A, 468, L57

Downes, D., \& Solomon, P. M. 1998, ApJ, 507, 615

Draine, B. T., Dale, D. A., Bendo, G., et al. 2007, ApJ, 663, 866

Dumouchel, F., Faure, A., \& Lique, F. 2010, MNRAS, 406, 2488

Dunne, L., Eales, S., Edmunds, M., et al. 2000, MNRAS, 315, 115

Dunne, L., \& Eales, S. A. 2001, MNRAS, 327, 697

Engel, H., Davies, R. I., Genzel, R., et al. 2010, A\&A, 524, A56

Engel, H., Davies, R. I., Genzel, R., et al. 2011, ApJ, 729, 58

Goldreich, P., \& Kwan, J. 1974, ApJ, 189, 441

Goldsmith, P. F., Langer, W. D., Pineda, J. L., \& Velusamy, T. 2012, ApJS, 203, 13

Graciá-Carpio, J., Sturm, E., Hailey-Dunsheath, S., et al. 2011, ApJL, 728, L7

Green, S., \& Thaddeus, P. 1976, ApJ, 205, 766

Greve, T. R., Papadopoulos, P. P., Gao, Y., \& Radford, S. J. E. 2009, ApJ, 692, 1432

Hildebrand, R. H. 1983, QJRAS, 24, 267

Imanishi, M., Nakanishi, K., Tamura, Y., Oi, N., \& Kohno, K. 2007, AJ, 134,2366

Iono, D., Wilson, C. D., Takakuwa, S., et al. 2007, ApJ, 659, 283

Klaas, U., Haas, M., Müller, S. A. H., et al. 2001, A\&A, 379, 823

Komossa, S., Burwitz, V., Hasinger, G., et al. 2003, ApJL, 582, L15

Lahuis, F., \& van Dishoeck, E. F. 2000, A\&A, 355, 699

Le Floc'h, E., Papovich, C., Dole, H., et al. 2005, ApJ, 632, 169

Luhman, M. L., Satyapal, S., Fischer, J., et al. 2003, ApJ, 594, 758

Malhotra, S., Kaufman, M. J., Hollenbach, D., et al. 2001, ApJ, 561, 766

Matsushita, S., Iono, D., Petitpas, G. R., et al. 2009, ApJ, 693, 56 
Max, C. E., Canalizo, G., \& de Vries, W. H. 2007, Sci, 316, 1877

Medling, A. M., Ammons, S. M., Max, C. E., et al. 2011, ApJ, 743, 32

Murray, N., Quataert, E., \& Thompson, T. A. 2005, ApJ, 618, 569

Nakanishi, K., Okumura, S. K., Kohno, K., Kawabe, R., \& Nakagawa, T. 2005, PASJ, 57, 575

Paggi, A., Fabbiano, G., Risaliti, G., Wang, J., \& Elvis, M. 2013, arXiv: 1303.2630

Paglione, T. A. D., Jackson, J. M., Ishizuki, S., \& Rieu, N.-Q. 1995, AJ, 109,1716

Planck Collaboration. 2011a, A\&A, 536, A21

Planck Collaboration. 2011b, A\&A, 536, A25

Rangwala, N., Maloney, P. R., Glenn, J., et al. 2011, ApJ, 743, 94

Sakamoto, K., Aalto, S., Wilner, D. J., et al. 2009, ApJL, 700, L104

Sakamoto, K., Scoville, N. Z., Yun, M. S., et al. 1999, ApJ, 514, 68

Sakamoto, K., Wang, J., Wiedner, M. C., et al. 2008, ApJ, 684, 957

Sanders, D. B., \& Mirabel, I. F. 1996, ARA\&A, 34, 749

Sanders, D. B., Scoville, N. Z., \& Soifer, B. T. 1991, ApJ, 370, 158

Sanders, D. B., Soifer, B. T., Elias, J. H., et al. 1988, ApJ, 325, 74

Schöier, F. L., van der Tak, F. F. S., van Dishoeck, E. F., \& Black, J. H. 2005, A\&A, 432, 369
Scoville, N. 2003, JKAS, 36, 167

Scoville, N., Aussel, H., Sheth, K., et al. 2014, ApJ, 783, 84

Scoville, N. Z., Evans, A. S., Dinshaw, N., et al. 1998, ApJL, 492, L107

Scoville, N. Z., Evans, A. S., Thompson, R., et al. 2000, AJ, 119, 991

Scoville, N., \& Murchikova, L. 2013, ApJ, 779, 75

Scoville, N. Z., Polletta, M., Ewald, S., et al. 2001, AJ, 122, 3017

Scoville, N. Z., \& Solomon, P. M. 1974, ApJL, 187, L67

Scoville, N. Z., Young, J. S., \& Lucy, L. B. 1983, ApJ, 270, 443

Scoville, N. Z., Yun, M. S., \& Bryant, P. M. 1997, ApJ, 484, 702

Semenov, D., Henning, T., Helling, C., Ilgner, M., \& Sedlmayr, E. 2003, A\&A, 410,611

Shirley, Y. L., Evans, N. J., II, Young, K. E., Knez, C., \& Jaffe, D. T. 2003, ApJS, 149,375

Spitzer, L., Jr. 1942, ApJ, 95, 329

Stacey, G. J., Hailey-Dunsheath, S., Ferkinhoff, C., et al. 2010, ApJ, 724,957

Tacconi, L. J., Genzel, R., Tecza, M., et al. 1999, ApJ, 524, 732

Tecza, M., Genzel, R., Tacconi, L. J., et al. 2000, ApJ, 537, 178

Thompson, T. A., Quataert, E., \& Murray, N. 2005, ApJ, 630, 167

Wilson, C. D., Rangwala, N., Glenn, J., et al. 2014, ApJL, 789, L36 